Des luttes étudiantes dans les années soixante en Europe Occidentale (Allemagne, France, Italie)

\title{
Herbert Marcuse, penseur de la révolte des étudiants allemands
}

Igor Krtolica

\section{OpenEdition}

Édition électronique

URL : http://journals.openedition.org/grm/282

DOI : $10.4000 / \mathrm{grm} .282$

ISSN : 1775-3902

Éditeur

Groupe de Recherches Matérialistes

Référence électronique

Igor Krtolica, « Herbert Marcuse, penseur de la révolte des étudiants allemands », Cahiers du GRM [En ligne], 3 | 2012, mis en ligne le 29 mai 2012, consulté le 30 avril 2019. URL : http:// journals.openedition.org/grm/282; DOI : 10.4000/grm.282 


\title{
Herbert Marcuse, penseur de la révolte des étudiants allemands
}

\author{
IGOR KRTOLICA
}

\begin{abstract}
L'idée de socialisme perd son caractère scientifique lorsque sa nécessité historique se dissout dans un avenir indéfini (et douteux). Les tendances objectives ne vont dans le sens du socialisme que dans la mesure où les forces subjectives qui luttent pour le socialisme réussissent à plier ces tendances dans le sens du socialisme, et à la faire maintenant : tout de suite, demain et dans les jours qui viennent.

- H. Marcuse, Contre révolution et révolte, 1972.-
\end{abstract}

Il sera question ici de l'analyse que fait Herbert Marcuse de la mutation du sujet révolutionnaire, c'est-à-dire des facteurs objectifs et subjectifs de la transformation sociale, et de la fonction qu'y prend la jeunesse étudiante dans les pays du capitalisme avancé. En guise de préambule, et afin de situer les enjeux de cette analyse pour et dans la conjoncture en question, nous pouvons faire trois séries de remarques.

Peut-on parler de " textes de conjoncture »? Si l'on tient compte du fil directeur général des recherches menées dans le cadre du GRM - «Penser (dans) la conjoncture »-quelques précautions doivent être prises quant au corpus marcusien. On sait que la production philosophique de Marcuse s'étale du début des années 1930 jusqu'à la fin des années 1970. L'appartenance de Marcuse à ce qu'on appelle l'École de Francfort (appartenance elle-même complexe et sujette à problèmes) ne peut jouer le rôle d'un principe unificateur a priori à même de garantir la possibilité de réduire les différents aspects de sa production à une seule et même entreprise théorique, continue et 
progressive. Il faut au contraire être attentif à la fonction des déplacements théoriques, des glissements sémantiques et des points d'inflexion qui constituent la matière même d'une pensée s'élaborant. Il ne peut être question de faire jouer entre eux des textes de diverses périodes sans faire de cette chronologie un élément même de l'analyse - et ce même sur la période des seules années 1950-1970, où Marcuse développe un freudo-marxisme marqué par une grande unité théorique et une profonde homogénéité conceptuelle. Pour ne citer que les deux ouvrages les plus connus, Éros et Civilisation (qui paraît en 1955) et L'homme unidimensionnel (1964): les neuf années qui séparent ces deux textes ne peuvent être tenues pour nulles. Mieux : à l'égard de ce qui nous occupe aujourd'hui, à savoir d'une part la manière dont une théorie se trouve transformée par une conjoncture (c'est-à-dire relativisée, limitée, ou bien validée et relancée) en même temps qu'elle cherche à se l'approprier (c'est-à-dire à inventer les concepts adéquats pour la penser), et d'autre part le diagnostic spécifique porté par Marcuse sur la révolte des étudiants allemands dans la seconde moitié des années 1960, il est a fortiori nécessaire de rester attentifs aux légers déplacements qui se manifestent dans chaque texte de cette période. De manière schématique, on pourrait diviser les textes de la période 1964-1975 en trois phases théoriques successives : $1^{\circ}$ L'homme unidimensionnel (1964) et Tolérance répressive (1964); $2^{\circ}$ les conférences et débats à la Freie Universität de Berlin-Ouest en 1967 réunis dans La fin de l'utopie (1968) et Vers la libération (1969); $3^{\circ}$ Contrerévolution et révolte (1973) et Actuels (1975). En schématisant un peu, on dira que: dans la première phase, Marcuse met en place les concepts fondamentaux de son analyse des sociétés industrielles avancées à partir des mécanismes de surrépression idéologiques et de la rationalité technologique (principe de rendement) qui y opèrent pour endiguer les potentialités révolutionnaires; dans la seconde, il infléchit son dispositif théorique pour intégrer dans la théorie l'émergence d'un nouveau facteur subjectif de la transformation sociale en fonction des forces subversives qui se manifestent exemplairement dans les révoltes estudiantines européennes et américaine et plus généralement de ce qu'on a appelé la Nouvelle Gauche (est emblématique à cet égard la préface de 1967 à L'homme unidimensionnel), ainsi que dans les luttes de libération nationale du Tiers Monde ; enfin, dans la troisième phase, Marcuse décale à nouveau le centre de gravité de ses analyses en raison de l'échec relatif de la Nouvelle Gauche à produire la révolution sociale 
escomptée. Si l'on adopte une telle périodisation interne pour cette portion du corpus marcusien, il faut du même coup renoncer à la tentation d'identifier des textes proprement conjoncturels au sein de son œuvre. Il n'est pas possible de distinguer des analyses conjoncturelles et des analyses que 1'on pourrait qualifier de structurelles, car l'exigence que la théorie soit en prise sur la conjoncture traverse l'ensemble de ces textes, elle les coupe diagonalement en obligeant constamment Marcuse à remettre ses thèses sur le métier. En effet, pour quelle raison pourrait-on refuser à chacun des textes de cette période le statut de texte de conjoncture ? Certes, les conjonctures ne sont pas les mêmes et ne sont pas ressenties avec la même intensité. À cet égard, bien que les textes de Marcuse ne soient pas centrés sur le cas allemand, il est très probable que la longueur et diversité des phases de la contestation étudiante à Berlin mais aussi aux États-Unis (versus la brièveté des événements parisiens de 1968 par exemple) ait joué un rôle non négligeable dans le caractère inchoatif de ses analyses.

Le freudo-marxisme comme niveau d'analyse. Avant d'entrer dans des considérations de détail sur le rapport de Marcuse à la révolte des étudiants allemands, je voudrais proposer l'hypothèse générale suivante : que l'efficace du freudo-marxisme de Marcuse ne tient peut-être pas tant à sa " vérité » (au sens de la validité objective de ses fondements) qu'au niveau d'analyse qui lui est propre, dans la mesure où il correspond à la situation spécifique de l'appareil universitaire dans le champ social des pays du capitalisme avancé, à savoir en bordure de l'appareil de production et du système idéologique. Ici même, Guillaume Sibertin-Blanc avait soulevé une

question d'époque, vigoureusement débattue dans les années 1960, qui tourne autour du problème de savoir si l'université est un appareil interne, ou du moins organiquement lié, à la structure de production dont elle forme les forces productives, ou si elle relève "seulement" d'un appareil de reproduction de rapports sociaux qui, même s'ils y sont liés, ne dérivent pas linéairement des rapports d'exploitation. ${ }^{1}$

S'appuyant sur la notion althussérienne d'Appareil Idéologique d'État (AIE) et sur le rôle singulier de l'AIE scolaire, il proposait de soutenir la thèse :

que l'appareil universitaire trouve justement sa spécificité d'être à la

${ }^{1}$ Cf. Séminaire du GRM : http://f.hypotheses.org/wpcontent/blogs.dir/1106/files/2013/01/GRM.3.Introduction..pdf. 
conjonction de la structure de production sociale et du système des rapports idéologiques et de leur reproduction, et que les conditions hétéronomes des antagonismes dont les étudiants seront les agents résident précisément dans le caractère irréductiblement problématique de cette conjonction où se condensent les contradictions de ces deux structures ${ }^{2}$.

Voici, enfin, comment il explicitait cette thèse :

l'AIE scolaire - et en son sein (sous des conditions spécifiques qu'il reste à déterminer) l'université comme institution de masse des États du "capitalisme avancé" - est donc dans une tension constitutive entre une structure économique à laquelle elle se rattache directement tout en en formant un appareil "séparé", relativement autonome par rapport au procès de production et aux conflits qui s'y développent (base objective de l'idéologie bourgeoise-républicaine de la "neutralité de l'école"), et un système idéologique dont elle fait partie tout en étant plus directement exposée que d'autres appareils idéologiques à subir les répercussions des contradictions internes aux rapports de production. ${ }^{3}$

Position de bordure, tension constitutive, conjonction problématique : différentes expressions pour qualifier la situation frontalière de l'appareil universitaire, entre la sphère économique et la sphère idéologique. Or, on sait que le succès du freudo-marxisme marcusien, avec Éros et civilisation et surtout L'homme unidimensionnel (dont je rappelle que le sous-titre est Essai sur l'idéologie dans la société industrielle avancée), repose sur une analyse unifiée et du procès de production et des formations désirantes-idéologiques qui lui sont liées : dimension économico-politique des complexes libidinaux, dimension libidinale de l'économie politique. Dans la préface de 1967 à L'homme unidimensionnel, après avoir insisté sur l'intrication du politique et du libidinal, Marcuse écrit à propos de la révolte estudiantine :

il est d'une importance qui dépasse de loin les effets immédiats que l'opposition de la jeunesse contre la "société d'abondance" lie rébellion instinctuelle et rébellion politique. (...) Ce qui est recherché ici - son élaboration conceptuelle n'est qu'au stade d'une lente gestation - est davantage et autre chose qu'une société fondée sur d'autres rapports de production (bien qu'une telle transformation de la base reste une condition nécessaire de la libération): il s'agit d'une société dans

\footnotetext{
${ }^{2}$ Ibid., p. 5 .

${ }^{3}$ Ibid., p. 8.
} 
laquelle les nouveaux rapports de production, et la production développée à partir d'eux, seront organisés par les hommes dont les besoins et les buts instinctuels seront la "négation déterminée" de ceux qui règnent dans la société répressive. ${ }^{4}$

Ainsi, en même temps que la théorie trouve son point de réalisation dans une pratique contestataire (lien de la rébellion instinctuelle et de la rébellion politique), une telle praxis se voit soutenue et comme justifiée par le dispositif conceptuel freudo-marxiste (comme niveau économique-libidinal d'analyse).

Marcuse et le cas des étudiants allemands. On sait que Marcuse quitte l'Allemagne 1933 pour les Etats-Unis, via la Suisse. Dans la période 19641975, le cas allemand n'est ni l'objet ni le centre de la plupart de ses analyses. Précaution majeure à prendre : on ne doit pas voir dans l'influence de Marcuse sur le mouvement étudiant (voire sur les mouvements étudiants européens) l'effet d'une adresse. C'est au contraire les étudiants qui ont érigé Marcuse en penseur de leur révolte : à Berlin, Rome ou Paris, on pouvait trouver les noms de Marx, Mao et Marcuse (les « $3 \mathrm{M} »)$ écrits côte à côte sur les murs ou les banderoles 5 . Les Italiens allèrent même jusqu'à forger le slogan « Marx est le Dieu, Marcuse son prophète et Mao le glaive $»^{6}$. Et quant au cas allemand, il existe un effet d'optique quant à l'appropriation des thèses de Marcuse par les étudiants berlinois en révolte, et notamment par Rudi Dutschke : c'est parce que Dutschke et consorts ont utilisé Marcuse pour penser leur situation et les

${ }^{4}$ Herbert Marcuse, L’homme unidimensionnel (1964), Paris, Minuit, 1968, Préface de 1967, p. 9.

${ }^{5}$ C'est Löwenthal qui apprend la nouvelle à Marcuse, cf. la lettre de Löwenthal à Marcuse du 16 août 1967, cité in H. MArcuse, Nachgelassene Schriften. Band 4: Die Studentenbewegung und ihre Folgen, Springe, zu Klampen Verlag, 2004, p. 186.

${ }^{6}$ Sur l'appropriation de Marcuse par les étudiants français, allemands et italiens, voir J.M. Palmier, Sur Marcuse, Paris, UGE, 1968, « V. Herbert Marcuse et la révolte des étudiants ». Cf. p. 71-172: «Le caractère étrange de l'influence [des thèses de Marcuse] sur la révolte mondiale des étudiants, c'est qu'il l'a à la fois prophétisée, inspirée et justifiée. On pourrait décrire un schéma commun à tous ces mouvements. Il s'agit toujours d'une contestation fondamentale, radicale de la société elle-même, de son idéologie et de son style de vie, qui est en apparence déclenchée par un malaise politique et social profond, ou une provocation violente des pouvoirs répressifs »; et Palmier de citer pêle-mêle la guerre du Vietnam et le malaise racial en Amérique ; le projet des lois d'exception, la politique corrompue de Bonn et le cynisme de la presse Springer en Allemagne; l'absence totale de liberté d'expression, l'ingérence de l'État dans la vie de chacun et l'interdiction d'une pièce de théâtre à Varsovie, l'occupation de la Sorbonne par les CRS et une crise sociale latente en France. 
moyens d'y réagir que celui-ci s'est trouvé, ex post, en être le théoricien - et non l'inverse. Ainsi, le diagnostic de Tolérance répressive n'a reçu un caractère éminemment conjoncturel ou actuel qu'en raison de son investissement politique direct par les étudiants au sein d'une actualité brûlante. Cependant, la dialectique faisant son œuvre, l'appropriation des thèses de Marcuse par Dutschke produit en retour un effet d'interpellation sur le philosophe exilé (sous la forme : «Hé, vous là-bas! le théoricien de la situation actuelle et des mouvements étudiants !»). Et cette interpellation a eu des conséquences concrètes : notamment la participation de Marcuse au Congrès sur le Vietnam à Francfort en mai 1966 (« Vietnam - L'analyse d'un exemple »), puis sa venue à l'Université Libre de Berlin-Ouest au début de l'été 1967 pour y tenir une série de conférences-débats (repris dans La fin de l'utopie). S'il y a donc eu adresse, elle a d'abord été dirigée vers Marcuse, et son effet s'est d'abord produit sur lui, l'adresse l'incitant à théoriser la conjoncture dont il est s'était retrouvé, presque malgré lui, le théoricien, fonction qu'il a par la suite, comme de plein gré, pleinement assumée. Néanmoins, bien que Marcuse ait suivi de près la révolte des étudiants allemands (du fait de cette adresse, mais aussi bien évidemment du fait de son origine allemande, de ses contacts avec Adorno et Dutschke, de sa venue régulière en RFA), celle-ci ne constitue quasiment jamais un objet d'analyse spécifique. À cet égard, le titre de ce texte («Herbert Marcuse et la révolte des étudiants allemands ») est déceptif : privilégiant le cas américain au sein des pays du capitalisme avancé, Marcuse théorise à peine la singularité du mouvement étudiant allemand face aux autres contestations estudiantines, mais l'englobe dans une analyse plus générale, et ce même quand il s'adresse aux étudiants de la F.U. en 1967. Ceci a deux conséquences pour notre propos : d'une part, il restera souvent, par la force des choses, à un certain niveau de généralité, qui n'obère certes pas le cas allemand mais qui l'englobe au sein d'un mouvement plus large; mais d'autre part, il est possible de percevoir en négatif la singularité de la révolte des étudiants allemands noyée dans les propos généraux de Marcuse, à condition d'être attentif au degré d'adéquation du cas allemand aux analyses générales, de faire parler les silences du texte et de tirer des conséquences particulières que Marcuse n'a pas lui-même tirées.

Venons-en maintenant aux analyses de ce qui constituait pour Marcuse la «situation actuelle » dans les années 1960 : la transformation profonde de 
l'objectivité du champ de l'économie politique des États du capitalisme avancé d'après guerre (liée en Allemagne au fameux « miracle économique ») et celle, corrélative, de la nouvelle subjectivité qui y émerge ; ou, pour le dire plus simplement, la double transformation des facteurs objectifs et subjectifs de la révolution, qui constitue l'horizon pratico-théorique des analyses de Marcuse. On en trouve une version synthétique dans l'article de 1968 intitulé « Réexamen du concept de révolution ${ }^{7}$. Avant de proposer une lecture plus détaillée des analyses de Marcuse à travers les différents textes que j'ai mentionnés plus haut et qui s'étalent de 1964 à 1975, en voici les éléments principaux tels qu'il les articule dans cet article. On peut les ramener à une série de trois thèses. $1^{\circ}$ Dans les pays du capitalisme avancé (dans les sociétés industrielles avancées), la classe ouvrière et ses organisations (syndicats et partis) ne constituent plus une force subversive totale, du fait de leur absorption et de leur neutralisation dans le système capitaliste et son idéologie (c'est l'acquis principal de L'homme unidimensionnel ${ }^{8}$ ) : la classe ouvrière demeure le facteur objectif de la transformation, mais elle n'en est plus un facteur subjectif. $2^{\circ}$ Dans ces mêmes pays est née une "solution de rechange " à l'égard des sociétés capitalistes mais également socialistes (la Nouvelle Gauche): cette nouvelle force d'opposition constitue le nouveau facteur subjectif et se caractérise par une concentration aux deux pôles de la société : elle réside dans la population des ghettos et dans l'élite intellectuelle d'origine bourgeoise (notamment les étudiants, mais aussi les mouvements féministes) ${ }^{9}$. $3^{\circ} \mathrm{Ces}$ deux groupes sont «extérieurs » au procès de production : dans les pays du centre qui nous occupent, ils ne peuvent devenir un facteur de changement qu'à condition d'être soutenus par la classe ouvrière (cf. le fameux problème de la rencontre entre étudiants et ouvriers, qui traverse l'ensemble des conjonctures de luttes étudiantes); autrement dit, la

${ }^{7}$ Cf. H. Marcuse, «Réexamen du concept de révolution », Diogène, $\mathrm{n}^{\circ} 64$ (oct-déc. 1968), Nouvelle actualité du marxisme, p. 21-32. On trouve également, en ouverture de ce même numéro, un article de Th. W. Adorno intitulé « Le marxisme est-il dépassé ? ».

${ }^{8}$ Cf. l'incipit de la Préface à l'édition française.

${ }^{9}$ Il pourrait paraître superflu de mentionner le Tiers Monde en plus des étudiants au sein des facteurs subjectifs de la transformation : on verra qu'il n'en est rien et que la solidarité possible entre les deux (cf. ce qu'Uwe Bergmann nommait le « semestre Vietnam » à Berlin) constitue pour Marcuse l'espoir le plus sérieux d'une transformation sociale globale (cf. Vers la libération, "Chapitre IV - La solidarité »). Cette hypothèse rejoint la notion d' "événements mondiaux intérieurs » que développait Guillaume Sibertin-Blanc dans la séance du 26 septembre du Séminaire du GRM (p. 23-27), cf. infra. 
transformation sociale qualitative ne peut intervenir qu'à condition d'une « synchronisation » entre les facteurs objectifs et subjectifs, d'une coïncidence et d'une catalyse des premiers par les seconds. Bien qu'elles soient inséparables et impliquent immédiatement les autres, ces trois thèses mutation de la classe ouvrière comme facteur objectif de la révolution, émergence de nouveaux facteurs subjectifs avec les étudiants et la population des ghettos, possibilité d'une coïncidence des deux facteurs comme condition nécessaire de la transformation - constitueront en quelque sorte les trois parties de ce texte.

\section{Facteur objectif : l'intégration de la classe ouvrière des pays du centre}

Comment la classe ouvrière en est-elle venue à ne plus constituer le facteur subjectif de la transformation sociale? Par quel «miracle» sa conscience de classe a-t-elle été idéologiquement soumise dans sa quasitotalité aux intérêts du mode de production capitaliste ? Quelle fonction peutelle dès lors continuer à assumer au sein du capitalisme avancé ? Herbert Marcuse, dans sa préface de 1967 à L'homme unidimensionnel, écrit :

J'ai analysé dans ce livre quelques tendances qui conduisent à une "société close" - close parce qu'elle met au pas et intègre toutes les dimensions de l'existence, privée et publique. Deux résultats de cette société sont d'une importance particulière : l'assimilation des forces et des intérêts oppositionnels dans un système auquel ils s'opposaient dans les étapes antérieures du capitalisme, et l'administration et la mobilisation méthodiques des instincts humains, ce qui rend ainsi socialement dirigeables et utilisables des éléments explosifs et "antisociaux" de l'inconscient. La puissance du négatif, largement incontrôlée aux stades du développement antérieur de la société, est maîtrisée et devient un facteur de cohésion et d'affirmation. ${ }^{10}$

Laissons pour plus tard ce qui constitue l'horizon le plus problématique (voire la tonalité apocalyptique) de cette citation, à savoir la profonde inquiétude que véhicule, pour l'hégélien invétéré qu'est Marcuse, la neutralisation du négatif dans les pays du capitalisme avancé : l'homogénéisation de l'hétérogène, la transformation de la différence qualitative en différence quantitative, le remplacement de la contradiction par

${ }^{10}$ H. Marcuse, L'homme unidimensionnel, op. cit., p. 7. 
la complicité, le passage du bidimensionnel à l'unidimensionnel, bref toutes les signes indiquant que le moteur de la dialectique est grippé... Nous en verrons la portée quant à l'espoir d'une transformation sociale. Quant aux deux tendances solidaires discernées par Marcuse - l'intégration de la classe ouvrière et la surrépression des instincts humains socialement subversifs -, elles sont l'effet de la transformation du mode de production capitaliste luimême, à laquelle renvoient les syntagmes de «capitalisme avancé » et de "société industrielle avancée », ou encore de "société d'abondance » et de « société de consommation ». Une telle transformation tient en grande partie au développement des forces productives, c'est-à-dire au rôle prépondérant pris par la technologie ou l'automation dans le procès de production capitaliste : c'est ce développement qui change la composition de la classe ouvrière (via l'augmentation des cols blancs et d'une classe moyenne) et permet l'asservissement de ses besoins (par le désir obsessif d'un meilleur niveau de vie). Dans les sociétés industrielles avancées, la classe ouvrière subit donc une double transformation : de sa composition et de sa conscience. En effet, la nouvelle composition de la classe ouvrière qui se traduit par la croissance proportionnelle des « cols blancs » face aux «cols bleus », par l'essor de ces nouvelles « classes moyennes » constituant désormais une large frange du prolétariat, produit une intégration de ses besoins. L'augmentation moyenne du niveau de vie général du prolétariat cesse de le rejeter hors du système et le conduit à désirer les bénéfices matériels qu'il paraît en mesure de réclamer et d'obtenir dans les conditions économiques florissantes qui caractérisent l'après-guerre. Cette double transformation de la classe ouvrière n'est rien d'autre, pour Marcuse, qu'une double scission: de l'unité de sa composition objective et de l'unité de sa conscience révolutionnaire. La « nouvelle classe ouvrière » est séparée de ce qu'elle est et de ce qu'elle peut.

Tandis que la proportion des «cols bleus » diminue constamment, les « cols blancs» (employés, techniciens, ingénieurs et spécialistes) gagnent sans cesse en nombre et en importance; il se crée par là des divisions internes dans la classe ouvrière. Ainsi, les couches de la classe qui ont subi de la façon la plus directe - et subissent toujours - la brutalité de l'exploitation, sont justement celles dont la fonction dans le processus de production devient aujourd'hui moins capitale; au contraire, l'intelligentsia joue dans ce processus un rôle de plus en plus décisif - intelligentsia instrumentaliste, mais intelligentsia tout de même. Grâce à sa position, il serait possible à cette "nouvelle classe 
ouvrière " de bouleverser le mode de production et les rapports de production, de les réorganiser et de leur donner une orientation nouvelle. Mais elle n'a pas intérêt à le faire, et n'en ressent pas le besoin de façon vitale : elle est bien rémunérée, et très intégrée au système. ${ }^{11}$

Il n'est pas possible ici de détailler les différents aspects qui définissent chez Marcuse cette transformation conjointe de la structure objective de classe et des positions subjectives de désir, dont L'homme unidimensionnel est tout entier l'étude. Pour couper au plus court, il est possible de focaliser l'analyse sur le niveau commun qui articule ces deux aspects en même temps qu'il en constitue l'issue potentielle et spécifique, à savoir la question de la technologie.

Une des particularités de l'œuvre de Marcuse au cours des années 19601970 réside en effet dans cette analyse de l'intégration de la classe ouvrière et de son potentiel subversif en fonction de la rationalité technologique qui commande l'évolution du mode de production capitaliste. Nous croyons qu'il est pertinent de voir dans la question du développement des forces productives, ou plus précisément dans la critique du « fétichisme des forces productives $»^{12}$, l'originalité de la position de Marcuse dans le champ de la théorie marxiste. Elle implique une série d'inflexions théoriques vis-à-vis de la conception marxiste classique de la révolution en tant qu'elle repose sur la simplification progressive de la contradiction au sein du mode de production capitaliste par le biais du développement des forces productives et de la baisse tendancielle corrélative du taux de profit ${ }^{13}$. Rejeter le développement des forces productives comme moteur de la transformation c'est tout bonnement rejeter l'économisme - latent mais diffus - du marxisme de l'époque. On sait certes que Marcuse n'est pas le seul à avoir critiqué l'économisme : à la même époque en France, à travers l'idée d'une surdétermination de la contradiction, Althusser rejette la notion idéaliste de contradiction héritée de Hegel, réforme la topique infrastructure-superstructure des formations sociales contre l'idée d'une distinction de l'essence économique et de l'apparence idéologique, et ouvre du même coup la porte à une nouvelle conception de l'efficace de l'idéologie dans la structure sociale. À sa manière, par l'existence et l'efficace réelle accordée à la superstructure et à la conjoncture, Althusser affranchit

${ }^{11}$ H. MARCuSE, Vers la libération, Paris, Minuit, 1969, p. 76-77.

${ }^{12}$ H. Marcuse, Contre-révolution et révolte (1972), Paris, Seuil, 1973, p. 11.

${ }^{13}$ Cf. K. MARX, Le Capital, III, Troisième section : « Loi de la baisse tendancielle du taux de profit ». 
donc la théorie marxiste de l'économisme qui la hantait ${ }^{14}$. C'est par un tout autre biais que Marcuse en vient à rejeter l'économisme : non pas par l'idée de surdétermination de la contradiction, mais par une analyse conjoncturelle du caractère directement idéologique pris par la technologie, comme le résume le syntagme de « rationalité technologique » : la technologie se voit investie d'un rôle répressif autrefois assumé par une violence explicite. Selon Marcuse :

l'originalité de notre société, écrit Marcuse, réside dans l'utilisation de la technologie plutôt que de la terreur, pour obtenir la cohésion des forces sociales dans un mouvement double, un fonctionnalisme écrasant et une amélioration croissante du standard de vie. ${ }^{15}$

À la base du malentendu sur la question du développement des forces productives se situe pour Marcuse une méconnaissance du rôle assumé par la technologie au niveau des positions subjectives : l'organisation technologique de la production économique n'est pas neutre du point de vue de la production désirante. De même que la structure du pouvoir d'État (l'appareil d'État centralisé et hiérarchisé dans ses fonctions) n'est pas une puissance neutre dont il suffirait de s'emparer pour la retourner et la faire fonctionner au bénéfice du prolétariat (c'est un des enjeux théoriques majeurs de la notion de dictature du prolétariat), de même l'appareil technologique est solidaire dans son fonctionnement d'une division sociale du travail oppressive qu'il reconduit (notamment l'opposition entre le travail manuel des cols bleus et le travail intellectuel des cols blancs). Il serait par conséquent contradictoire de vouloir transformer les rapports sociaux de production sans toucher au procès technologique qui en garantit la stabilité et la relative efficacité, qui non seulement en reproduit les contradictions mais en reproduit constamment les conditions. L'accroissement quantitatif des forces productives ne peut donc donner lieu à une transformation qualitative de la société : dans les termes du livre III du Capital, le règne de la liberté ne peut être établi sur la base du règne de la nécessité, mais en transformant le règne de la nécessité lui-même,

${ }^{14}$ Cf. L. Althusser, Pour Marx (1965), «Contradiction et surdétermination » (1962), Paris, La Découverte, 2005, p. 113 : «jamais la dialectique économique ne joue à l'état pur, (...) jamais dans l'Histoire on ne voit ces instances que sont les superstructures, etc., s'écarter respectueusement quand elles ont fait leur œuvre ou se dissiper comme son pur phénomène pour laisser avancer sur la route royale de la dialectique sa majesté Économie parce que les Temps seraient venus. Ni au premier, ni au dernier instant, l'heure solitaire de la "dernière instance" ne sonne jamais ».

${ }^{15}$ H. MARCuSE, L'homme unidimensionnel, op. cit., p. 16. 
c'est-à-dire la fonction qu'y prennent les forces productives. La transformation sociale, pour échapper aux illusions du socialisme scientifique, doit briser la rationalité technologique qui assure la continuité et en définitive la solidarité profonde entre les pays capitalistes et socialistes. Remettre en question la fonction de l'appareil technologique non seulement au sein du procès de production mais à l'échelle de la société toute entière, c'est remettre en question la rationalité technologique qui y préside sous la forme du principe de rendement :

Il s'agit d'une totale conversion des valeurs, d'une transformation des besoins et des objectifs visés, qui implique encore un autre changement dans le concept de la révolution : une rupture dans la continuité de l'appareil technique de la productivité qui, selon Marx, expurgé des abus du capitalisme se prolongerait dans la société communiste. Une telle continuité technologique, si elle devait être envisagée, constituerait un enchaînement fatal (plutôt qu'une rupture) entre le capitalisme et le socialisme, pour la simple raison que cet appareil technique est devenu, dans sa structure même et dans sa portée, un appareil d'asservissement et de domination. ${ }^{16}$

La critique de l'économisme chez Marcuse est naturellement solidaire d'une réinterprétation du rôle de la paupérisation quant à la radicalisation de la contradiction de classe : elle ne suffit plus (si tant est que cela fût jamais le cas) à produire une conscience prolétarienne ; inversement, sa suppression ne peut suffire à caractériser le socialisme puisque celui-ci passe par la définition de nouveaux besoins et non pas leur simple satisfaction.

Il reste vrai que le développement du capitalisme passe par une paupérisation croissante, et que la paupérisation sera un facteur fondamental de la révolution - mais sous de nouvelles formes historiques. Dans la théorie de Marx, la paupérisation voulait tout d'abord dire privation, insatisfaction de besoins vitaux, et avant tout de besoins matériels. Cette notion ayant cessé de représenter la condition des classes ouvrières dans les pays industriels de pointe, on l'a réinterprétée pour y voir une frustration relative, un appauvrissement culturel par rapport à la richesse sociale disponible. Mais cette réinterprétation suggère une continuité fallacieuse dans la transition vers le socialisme, à savoir l'amélioration de la vie dans le cadre de l'univers existant des besoins. Alors que ce qui est en jeu dans la révolution

${ }^{16}$ H. MARCuSE, « Réexamen du concept de révolution », op. cit., p. 29. 
socialiste, ce n'est pas la seule généralisation de la satisfaction à l'intérieur de l'univers existant des besoins, ni le déplacement de la satisfaction d'un certain niveau à un niveau supérieur, mais la rupture avec cet univers, le saut qualitatif. La révolution implique une transformation radicale des besoins eux-mêmes et des aspirations, tant culturelles que matérielles; de la conscience et de la sensibilité ; du processus du travail aussi bien que du loisir. ${ }^{17}$

La relativisation du critère de la paupérisation comme facteur économique-libidinal d'une conscience révolutionnaire permet d'expliquer simultanément deux phénomènes. D'une part que les classes ouvrières des sociétés industrielles avancées ne soient plus un facteur subjectif de la transformation sociale puisque le système assure plus que la simple reproduction de la force de travail : la conscience de l'exploitation varie en raison inverse de la satisfaction des besoins immédiats et de l'intégration au système. Mais d'autre part, le fait que la paupérisation ne joue plus un rôle prépondérant quant à la conscientisation des masses permet a contrario d'éclairer le caractère le plus singulier des révoltes étudiantes dans ces mêmes sociétés florissantes : que des futures élites intellectuelles qui bénéficient des richesses d'un capitalisme prospère entrent malgré tout en révolte contre lui. Comment expliquer que des révoltes aient lieu dans les pays du centre et de la part des fractions les plus intégrées de la société sinon en réévaluant le rôle des facteurs idéologiques au détriment des seules composantes économiques ? Le nouveau stade atteint par le capitalisme, les nouvelles conditions objectives de la production, de la circulation et de la consommation impliquent que la transformation sociale doit être désirée en fonction de revendications et de facteurs subjectifs inédits, qui s'opposent à la société non pas tant par la misère mais par la prospérité aliénante qu'elle engendre.

La fonction que Marcuse accorde à la technologie ne fait pas simplement l'objet d'une dénonciation. Il ne se contente pas de rejeter le fétichisme économiste des forces productives en y décelant l'effet illusoire

${ }^{17}$ H. MARCuSE, Contre-révolution et révolte, op. cit., p. 27-28. Sur ce point, voir également H. Marcuse, ibid., p. 11 : «Le socialisme doit augmenter la quantité des biens et services disponibles afin de supprimer toute pauvreté, mais la production socialiste doit en même temps changer la qualité de l'existence: changer les besoins eux-mêmes et leur satisfaction. Les facultés morales, psychologiques, esthétiques et intellectuelles que l'on relègue aujourd'hui en un domaine culturel séparé de l'existence matérielle et placé au-dessus d'elle quand elles ne sont pas complètement étiolées - deviendraient alors autant de facteurs de la production matérielle elle-même ». 
d'une rationalité technologique. Car la technologie n'est pas uniquement ce qui nous fait rester dans l'orbite du principe de rendement capitaliste, elle est aussi, paradoxalement - ou plutôt dialectiquement -, ce qui peut nous en fait sortir. En effet, le développement proprement capitaliste des forces productives produit des besoins que le mode de production capitaliste luimême ne peut pas satisfaire. La dynamique capitaliste est bel et bien contradictoire en tant qu'elle produit les conditions de sa propre abolition. Aussi la société d'abondance que décrit Marcuse depuis les années 1950 estelle éminemment ambivalente : si d'un côté elle unidimensionnalise la réalité, intègre les éléments négatifs ou transcendants qui en produisaient la critique effective (notamment la classe ouvrière et ses organisations représentatives : syndicats et partis marxiste-léniniste, tant dans leurs revendications objectives que dans leurs positions subjectives), elle suscite néanmoins de l'autre côté de nouveaux éléments négatifs ou transcendants que la pensée critique tâche de dégager (c'est tout le problème de la «pensée unidimensionnelle » dans L'homme unidimensionnel : quelle place reste-t-il à une pensée négative $?^{18}$ ). La production en abondance de biens de consommation, l'élévation générale du niveau de vie met le système capitaliste en demeure de répondre aux nouveaux désirs de consommation qu'il produit, dont sa survie dépend en même temps qu'ils la menacent.

Cette force assimilatrice reste une force de désintégration. L'organisation totale de la société soumise au capitalisme de monopoles et l'opulence croissante qu'elle crée ne peuvent ni inverser ni bloquer la dynamique de son expansion: le capitalisme ne peut satisfaire les besoins qu'il engendre. L'élévation même du niveau de vie exprime cette dynamique: elle a obligé à susciter sans cesse de nouveaux besoins qui puissent être satisfaits sur le marché; elle provoque maintenant des besoins transcendants dont la satisfaction impliquerait l'abolition du mode de production capitaliste. (...) Le lieu historique de la révolution est précisément le stade de développement à partir duquel la satisfaction des besoins vitaux crée d'autres besoins qui transcendent les sociétés capitalistes d'État et socialiste d'État. (...) Les forces centrifuges qui apparaissent avec l'émergence de besoins transcendants prennent les manipulateurs capitalistes à revers et c'est le mode de

${ }^{18} \mathrm{Cf}$. H. MARCUSE, L’homme unidimensionnel, « La pensée unidimensionnelle ». Voir également, p. 251 : «La pensée critique doit s'efforcer de définir le caractère irrationnel de la rationalité établie (ce qui devient de plus en plus évident) et de définir les tendances qui poussent cette rationalité à engendrer sa propre transformation ». 
production lui-même qui en est la source. (...) Le progrès technique et l'offre considérable de "luxes» créent et recréent, parallèlement au monde du travail aliéné, dans la publicité quotidienne et les éclatants signes extérieurs de l'opulence matérielle, les images d'un monde qui ne serait plus le privilège exclusif d'une élite et semble à la portée des masses. Les succès techniques du capitalisme débouchent sur un univers de frustration, de manque de bonheur et de répression. Le capitalisme a dessiné une nouvelle dimension qui est tout à la fois et simultanément son propre espace vital et sa négation. ${ }^{19}$

Le développement contradictoire des forces productives produit donc deux effets simultanés au sein du système capitaliste : il en constitue la menace en même temps qu'un puissant motif d'espérer puisqu'il atteint un stade qui est « le lieu historique de la révolution », bien que celui-ci réponde à « des incitations radicalement nouvelles $»^{20}$. Une nouvelle forme de révolution paraît désormais possible : révolution culturelle, qui prend en charge toutes les composantes de la civilisation, économico-politiques et idéologiqueslibidinales. C'est précisément à ce niveau de la réflexion de Marcuse que le cas des révoltes étudiantes reçoit une signification spécifique. Dans L'homme unidimensionnel et dans Tolérance répressive, qui datent tous deux de 1964, Marcuse décèle la potentialité révolutionnaire objective à l'œuvre dans la dynamique capitaliste mais n'y voit aucun indice d'une réappropriation subjective à venir. Il ne fait qu'y constater un inquiétant écart entre la théorie et la pratique, entre les processus objectifs que la pensée critique théorise et l'absence de forces subjectives susceptibles de les investir idéologiquement ce qui remet en question le schéma traditionnel de la révolution non moins que son éventualité.

Le tout a beau montrer avec évidence qu'il est irrationnel, qu'un changement est nécessaire, cela ne suffit pas pour provoquer un changement; il ne suffit pas de comprendre que le changement est nécessaire pour rendre possible une évolution différente. (...) Est-ce que cela veut dire que la théorie critique doive abdiquer et abandonner le terrain à la sociologie empirique (...) ? Ou les concepts dialectiques témoignent-ils encore une fois de leur vérité - permettent-ils de comprendre que leur propre situation est celle de la société qu'ils analysent ? La réponse est toute prête : il suffit pour la connaître de

${ }^{19}$ H. Marcuse, Contre-révolution et révolte, op. cit., p. 27-31.

${ }^{20}$ Ibid., p. 30. 
considérer la théorie critique du point précisément de sa plus grande faiblesse - elle est incapable de démontrer qu'il y a des tendances libératrices à l'intérieur de la société établie. (...) Tant que les forces réelles sociales n'ont pas fait leurs preuves, la critique de la société est encore valable et rationnelle, mais elle est incapable de traduire sa rationalité en termes de pratiques historiques. Que faut-il conclure? La «libération des éventualités inhérentes"n'exprime plus de façon adéquate une évolution historique possible. ${ }^{21}$

L’idée que la « libération des éventualités inhérentes » n'exprime plus une évolution historique possible ne signifie rien d'autre que : la classe ouvrière ne peut plus être à elle seule le sujet de l'émancipation. Ce que viendront confirmer l'émergence des luttes étudiantes et leur analyse immédiate.

\section{Facteurs subjectifs : des nouvelles forces subversives}

Dès 1967, Marcuse prend conscience de la portée inédite des révoltes étudiantes aux États-Unis et en RFA. Loin de considérer ces mouvements de contestation comme de simples troubles émanant des futures élites de la société, loin de les envisager comme une vulgaire agitation de surface dont les revendications seraient détachées des problèmes économiques réels et flotteraient dans la nébuleuse idéologique de l'ordre du désir - et que la théorie marxiste n'aurait dès lors pas à prendre au sérieux -, Marcuse perçoit très tôt que ces révoltes questionnent le dispositif théorique marxiste dans sa structure et dans ses concepts. En témoignent les textes des conférences et débats tenus à l'Université Libre de Berlin-Ouest au mois de juillet 1967 et réunis dans La fin de l'utopie, ainsi que Vers la libération qui se présente comme une suite de L'homme unidimensionnel.

Marcuse y reprend l'idée que la classe ouvrière n'est plus le sujet de la révolution dans les sociétés industrielles avancées puisqu'elle est objectivement scindée (du fait de la transformation de sa composition) et subjectivement aliénée (par les besoins issus la société d'abondance) ${ }^{22}$. Mais il

${ }^{21}$ H. MARCuSE, L'homme unidimensionnel, op. cit., p. 277-278 (je souligne).

${ }^{22}$ Sur l'absentement du sujet révolutionnaire dans les pays capitalistes du centre, voir la déclaration de Marcuse en 1964 dans Tolérance répressive : «On ne peut identifier les forces d'émancipation à aucune classe sociale qui, en vertu de ses conditions matérielles, serait libre de 
y ajoute une idée décisive : est en train d'émerger une nouvelle conscience révolutionnaire en dehors de la classe ouvrière. Reprenant l'idée d'un écart entre théorie et pratique, entre facteurs objectifs et subjectifs de la transformation sociale, entre puissance et conscience révolutionnaires, il adjoint ainsi l'hypothèse que la jeunesse étudiante constitue une nouvelle conscience répondant aux transformations objectives du champ social des sociétés capitalistes avancées. La thèse marcusienne de l'émergence d'un nouveau sujet de l'émancipation est donc adossée à une analyse des conditions objectives inédites qui caractérisent le stade avancé du capitalisme d'après guerre. Ce repérage des facteurs subjectifs par Marcuse renvoie à la transformation des conditions objectives, et ne relève en rien d'une simple projection fantasmatique. Or, la transformation des conditions objectives entraîne une modification nécessaire des catégories traditionnelles de la théorie marxiste du sujet révolutionnaire : car celui-ci n'est plus localisé ni localisable dans le prolétariat mais émane d'autres couches de la population, qui entretiennent avec la classe ouvrière des relations qui ne sont pas de simple subordination et dont la composition y est radicalement irréductible.

Pour la théorie marxiste, la localisation - ou plutôt, la concentration de l'opposition dans certaines couches moyennes et dans la population des ghettos apparaît comme une intolérable déviation; de même que l'accent qui est mis sur les besoins biologiques et esthétiques, et où l'on croit voir un retour à l'idéologie bourgeoise, ou même, pis encore, à l'idéologie féodale. Pourtant, dans les pays avancés où règne le capitalisme des monopoles, ce déplacement de l'opposition, ce transfert du rôle des organisations ouvrières à des minorités militantes est l'effet du développement interne de la société, et la prétendue "déviation » théorique ne fait que refléter ce développement. (...) De ce point de vue, le fait que les forces négatrices se soient éloignées de leur base traditionnelle (dans les couches opprimées), loin de signifier que l'opposition résiste mal à la capacité d'intégration du capitalisme avancé, exprime peut-être qu'une base nouvelle se constitue peu à peu, faisant apparaître le nouveau Sujet historique du changement, dont les besoins et les aspirations, dans leur différence qualitative, répondent aux

toute fausse conscience. Aujourd'hui, elles sont désespérément dispersées dans toute la société » (H. Marcuse, Tolérance répressive (1964), Paris, Homnisphères, 2008, p. 72). C'est seulement à la faveur de la naissance des contestations étudiantes, d'abord aux États-Unis puis en Allemagne et en France, que Marcuse redonnera une consistance objective au sujet de l'émancipation (cf. infra). 
nouvelles conditions objectives. ${ }^{23}$

Crise des catégories traditionnelles de la théorie marxiste : aux nouvelles conditions objectives correspondent de nouvelles forces subjectives que la pensée critique doit repérer. D'où une double question qui traverse l'ensemble des textes de Marcuse à ce sujet. Qu'advient-il, d'une part, du rôle de la classe ouvrière quant à la révolution ? Et d'autre part, quel est ce nouveau sujet historique du changement et quelle fonction assume-t-il vis-àvis de l'éventualité d'une transformation sociale?

Que Marcuse ait immédiatement accordé une grande importance aux mouvements étudiants ne doit pas laisser penser qu'il a évacué tout aussi prestement le rôle traditionnellement dévolu à la classe ouvrière et à ses organes représentatifs (parti et syndicats) quant au projet révolutionnaire. D'abord, on le verra, parce que les mouvements étudiants appartiennent à la tendance plus profonde et plus large de la Nouvelle Gauche, dont ils ne sont qu'un aspect, et parce que de tout autres forces subversives existent dans le monde capitaliste. Ensuite, parce que la classe ouvrière conserve à ses yeux son attribut historique principal: celui d'être la puissance de masse sans laquelle aucune révolution authentique n'est possible. Néanmoins, sa conscience politique n'est plus (ou pas encore) révolutionnaire : c'est pourquoi le diagnostic de Marcuse porte moins sur la classe ouvrière ellemême que sur les facteurs subjectifs susceptibles d'actualiser sa puissance révolutionnaire virtuelle.

La transformation radicale d'un système social dépend, aujourd'hui encore, de la classe qui constitue la base humaine du processus de production ; c'est-à-dire, dans les pays capitalistes avancés, de la classe ouvrière. La composition de cette classe, ainsi que son degré d'intégration au système, ont subi un changement qui modifie, sinon son rôle virtuel, du moins son rôle politique immédiat. Classe révolutionnaire « en soi » mais non « pour soi», objectivement mais pas subjectivement, sa radicalisation dépendra de catalyseurs « extérieurs » à elle. ${ }^{24}$

Le fait majeur de l'après-guerre dans les sociétés industrielles avancées n'est pas uniquement l'intégration de la classe ouvrière au système capitaliste via l'amélioration du niveau de vie, la modification de sa composition et la

${ }^{23}$ H. MARCUSE, Vers la libération, op. cit., p. 73-74.

${ }^{24}$ Ibid., p. 75. 
transformation idéologique et répressive de ses «besoins» et désirs. C'est également la neutralisation des partis marxistes-léninistes dont la fonction consistait au moins depuis Lénine à catalyser la conscience révolutionnaire des masses prolétariennes, à opérer ce fameux - mais épineux voire aporétique - passage de l'en-soi au pour-soi. En Allemagne, le SDS a été particulièrement sensible à cette intégration, et ce d'autant plus nettement qu'il est exclu du SPD en novembre 1961, parti qu'il considérera dorénavant comme appartenant à la Vieille Gauche. Le SDS - écrit Kohser-Spohn :

voyait le SPD comme un organe constitutionnel participant à la répression bureaucratique au même titre que toutes les autres institutions capitalistes. (...) Si la critique du SDS se dirigeait contre le SPD, elle ne critiqua pas moins le caractère d'intégration, de dépolitisation et de manipulation d'autres organes ouvriers, tels que les syndicats ou les organisations de type bolchevique. ${ }^{25}$

La neutralisation des organes représentatifs de la classe ouvrière (partis communistes et syndicats) est elle-même l'effet du nouveau stade objectif du capitalisme et de la situation géopolitique de guerre froide imposant un relatif équilibre des forces : d'où leur intégration au jeu démocratique du dialogue et de la négociation et leur parlementarisation.

Le développement d'une conscience politique radicale dans les masses n'est concevable que lié à un affaiblissement de la stabilité économique et de la cohésion du système. Tel était le rôle traditionnel du parti marxiste-léniniste : préparer le terrain à ce développement. La capacité de stabilisation et d'intégration du capitalisme avancé, et les impératifs de la «coexistence pacifique», ont contraint ce parti à se " parlementariser », à s'intégrer au processus démocratique bourgeois, à se concentrer sur des revendications de nature économique, de sorte que, loin de promouvoir la croissance d'une politique radicale, il contribue bien plutôt à l'inhiber. Là où est apparue une telle conscience à l'intérieur de l'appareil du parti et des syndicats, ç'a été le fait de forces «extérieures», issues principalement de l'intelligentsia; l'appareil n'a suivi le mouvement que lorsqu'il commençait à acquérir de la vitesse, et dans l'unique dessein d'en reprendre le contrôle. ${ }^{26}$

Comment Marcuse explique-t-il que l'apparition d'une conscience

${ }^{25}$ C. Kohser-Spohn, Mouvement étudiant et critique du fascisme en Allemagne dans les années soixante, Paris, L'Harmattan, 1999, p. 63-64.

${ }^{26}$ H. MARCuSE, Vers la libération, op. cit., p. 76. 
révolutionnaire qui faisait défaut aux dirigeants des organes politiques de la classe ouvrière ait émané en large partie de l'intelligentsia, et notamment des jeunesses étudiantes ? Conformément à ce qu'affirme Guillaume SibertinBlanc, il n'y a pas de hasard là-dedans, et Marcuse ne s'y trompe pas : une telle localisation tient principalement à la position spécifique de l'Université dans le champ social, à l'articulation de l'économique et de l'idéologique. La nouvelle subjectivité révolutionnaire est tendanciellement le fait de 《 marginaux improductifs $»^{27}$, qui constituera à la fois la force et la faiblesse des mouvements politiques qui en sont issus.

L'intégration crée des conditions telles que le besoin vital d'un changement radical ne donne naissance à une nouvelle conscience politique que dans des groupes sociaux qui, pour des raisons objectives, sont (relativement) libres par rapport aux aspirations et aux intérêts conservateurs sur lesquels repose l'intégration : libres de procéder à une transmutation radicale des valeurs. ${ }^{28}$

Marcuse n'identifie pas «le» nouveau sujet révolutionnaire (aussi divers ou éclaté soit-il), mais dégage des tendances dont l'existence est par définition problématique. Il n'y a donc pas pure et simple substitution des marginaux improductifs au prolétariat: non seulement parce que la classe ouvrière conserve son statut de classe révolutionnaire en soi, mais aussi parce que cette transformation modifie la nature même de ce qu'on appelle " sujet révolutionnaire ». La continuité nominale ne doit pas masquer une rupture dans la théorie comme dans la pratique : la possibilité d'assigner un sujet unique de l'émancipation et les modes d'organisation et de luttes qui lui étaient corrélatifs ont disparu ${ }^{29}$. La nouvelle opposition que tendent à

${ }^{27}$ H. MARCuSE, Contre-révolution et révolte, op. cit., p. 50.

${ }^{28}$ H. MARCuSE, Vers la libération, op. cit., p. 76.

${ }^{29}$ Cf. H. Marcuse, La fin de l'utopie, Paris, Seuil, 1968, p. 18 : « Si Marx a vu dans le prolétariat la classe révolutionnaire, il l'a fait aussi, et peut-être même d'abord, parce que le prolétariat était exempt des besoins répressifs de la société capitaliste, parce que dans le prolétariat les nouveaux besoins de liberté pourraient se développer et n'étaient pas étouffés par les anciens besoins répressifs. Aujourd'hui ce n'est plus le cas dans la plus grande partie des pays capitalistes avancés. La classe ouvrière ne porte plus en elle la négation des besoins régnants. C'est l'un des faits les plus sérieux que nous devions affronter. En ce qui concerne maintenant les forces de transformation elles-mêmes, j'admets volontiers que personne $n$ 'est aujourd'hui en mesure de nous donner une recette et de nous dire: voici les forces révolutionnaires, voici ce qu'elles sont en mesure d'accomplir, voici ce qu'il faut faire » (nous soulignons). Mais inversement, on pourrait légitimement penser que la nouvelle situation à 
constituer ces " minorités » (au sens où l'entend Guillaume Sibertin-Blanc dans ses «Éléments de problématisation pour une analyse des processus de politisation étudiante $»$ ), et notamment celles en prise sur la théorie et l'idéologie que sont les étudiants, ne relègue pas la classe ouvrière au musée des antiquités du marxisme : elle lui confère une fonction inédite en même temps qu'elle interroge la possibilité de son articulation aux nouveaux modes de luttes (articulation dont une des types est connue sous la forme de la rencontre - souvent « manquée » - entre étudiants et ouvriers).

Il est évidemment aberrant de prétendre que l'opposition des classes moyennes est en train de remplacer le prolétariat dans sa fonction de classe révolutionnaire, et que le Lumpenproletariat devient une force politique radicale. En réalité, on assiste à la constitution de groupes, encore relativement restreints et faiblement (souvent même pas du tout) organisés, dont la conscience et les besoins servent de catalyseurs à la révolte, à l'intérieur des majorités auxquelles ces groupes appartiennent par leur origine de classe. En ce sens, l'intelligentsia militante est évidemment coupée des classes moyennes, comme la population des ghettos est coupée des organisations ouvrières. ${ }^{30}$

L'émergence de nouvelles forces subversives ne résout pas le problème que semblait poser la neutralisation des organes politiques de la classe ouvrière. Les étudiants n'endossent pas le costume et le rôle des dirigeants communistes ou syndicaux, pas plus que la population des ghettos ne remplace le prolétariat. Les mêmes fonctions ne subsistent pas derrière le changement des agents, d'abord et avant tout du fait de la position marginale de ces forces subversives. Elles se situent aux deux pôles opposés de la société : du côté des élites pour les étudiants, du côté du Lumpenproletariat pour les populations des ghettos ${ }^{31}$. La nature de la scission entre la base

laquelle fait face la théorie marxiste après la Seconde Guerre mondiale, que l'éclatement et la dispersion réels du sujet de l'émancipation ne fait que révéler rétrospectivement que celui-ci n'a en réalité jamais été ni un ni stable, bien qu'il ne l'ait pas été d'une tout autre manière (ceci pour ne pas méconnaître le changement effectivement intervenu autour de la Seconde Guerre mondiale). La fameuse distinction entre classe révolutionnaire en soi et pour soi, moteur de la politique marxiste-léniniste, serait dès lors moins l'occasion d'un passage que l'indice d'un irréductible écart - celui dans lequel toute politique marxiste serait amenée à se loger (cf. infra, les remarques conclusives).

${ }^{30}$ H. MARCUSE, Vers la libération, op. cit., p. 72.

${ }^{31} \mathrm{Cf}$. H. MARCUSE, « Réexamen du concept de révolution », op. cit., p. 25-26: « Au centre du capitalisme corporatif, l'opposition se caractérise par une concentration aux deux pôles de la 
objective et la conscience subjective est donc tout autre que dans le cas du rapport entre le parti et la masse : le rapport entre étudiants et population des ghettos d'une part et classe ouvrière intégrée d'autre part inaugure une nouvelle forme d'écart ou de scission, de sorte que le problème ne se pose pas en termes de représentation et de conscientisation : les étudiants ne constituent pas l'avant-garde du prolétariat ${ }^{32}$. (Dans la suite, nous ne tiendrons pas compte de la population des ghettos, qui exprime ici le point de vue américano-centré des analyses de Marcuse et fait en l'occurrence référence aux luttes des minorités raciales aux États-Unis et notamment au mouvement des droits civiques; nous ne croyons pas qu'il y ait de réel équivalent en Allemagne à la même époque, ce qui ne remet toutefois pas en question l'idée que les nouveaux facteurs subjectifs naissent en marge du procès de production).

Les mouvements étudiants de la fin des années 1960, et notamment la révolte des étudiants allemands, nourrissent un nouvel espoir de révolution en même temps qu'ils provoquent une nouvelle crainte : que l'écart entre facteurs objectifs et facteurs subjectifs de la révolution demeure irréductible.

Dans la zone capitaliste, les deux facteurs historiques de la transformation, le facteur objectif et le facteur subjectif, ne coïncident pas: ils s'incarnent dans des groupes sociaux différents, voir antagonistes. Le facteur objectif, c'est-à-dire la base humaine du processus de production par lequel se perpétue la société établie, apparaît dans la classe ouvrière industrielle, source et réservoir de l'exploitation; et le facteur subjectif, c'est-à-dire la conscience politique, dans la jeune intelligentsia non conformiste ; enfin, le besoin de changement comme besoin vital constitue l'existence même de la population des ghettos - et aussi, dans les pays capitalistes peu avancés, des fractions « sous-privilégiées » de la classe ouvrière. ${ }^{33}$

La théorie marxiste-léniniste traditionnelle donnait à voir un sujet révolutionnaire marqué par une scission interne entre l'en-soi et le pour-soi. Le sujet de la révolution (la classe ouvrière) devait accéder à la conscience de

société : d'une part, dans la population des ghettos (population qui est elle-même loin d'être homogène) ; d'autre part dans l'élite intellectuelle d'origine bourgeoise, et plus particulièrement chez les étudiants. (...) Le fait que cette opposition ne figure pas dans le schéma de "l'orthodoxie marxiste" est en soi caractéristique de la structure du capitalisme corporatif ("intégration" de la majorité de la population soumise à la domination des grandes "corporations") ».

${ }^{32}$ Sur ce point, cf. H. MARCuSE, Contre-révolution et révolte, op. cit., p. 61-62.

${ }^{33}$ H. MARCuse, Vers la libération, op. cit., p. 78. 
soi par la médiation interne d'un parti ouvrier constitué d'une avant-garde prolétarienne censée opérer le passage de l'universel abstrait à l'universalité concrète et devenue $\mathrm{e}^{34}$. Or, pour Marcuse, la scission n'est désormais plus interne mais externe : elle ne passe plus entre des éléments d'un même groupe social, mais entre différents groupes sociaux incarnant chacun une partie du sujet révolutionnaire. Aucune partie n'est une condition suffisante de la transformation sociale : la base ouvrière constitue la force objective, une masse majoritaire mais passive, tandis que les étudiants et la population des ghettos constituent la force subjective, une conscience active dénuée de puissance pratique. Bref, la classe ouvrière est une force sans conscience, le mouvement étudiant une conscience sans force.

Révolutionnaire par sa théorie, par ses instincts, par les buts derniers qu'il se propose, le mouvement étudiant n'est pas une force révolutionnaire, ni peut-être même une avant-garde aussi longtemps qu'il n'y aura pas de masses capables et désireuses de le suivre ; mais il est le ferment de l'espoir, face à la toute-puissance du capitalisme et l'ambiance étouffante qui règne dans les métropoles capitalistes : il témoigne de la réalité de l'alternative. ${ }^{35}$

Du fait de l'impuissance native des mouvements étudiants, Marcuse s'est toujours refusé à les considérer comme des révolutions. Il qualifie constamment ces luttes de « révoltes », jamais de « révolution ». C'est ce qu'il déclare clairement aux étudiants de l'Université Libre de Berlin-Ouest en juillet 1967 et c'est ce sur quoi repose la spécificité de son analyse des luttes étudiantes en même temps que leur incomplétude: pour devenir révolutionnaires, ces luttes exigent une organisation et un relais dans la majorité ouvrière de la population puisque leur puissance de désintégration est insuffisante.

Vous savez peut-être que parmi les nombreuses choses qui m'ont été reprochées, il y en a deux qui ont récemment attiré une attention particulière. Premièrement, j'aurais affirmé que l'opposition étudiante en tant que telle est capable aujourd'hui déjà de faire la révolution.

${ }^{34}$ Pour une discussion du rôle du parti et de son avant-garde dans la prise de conscience de classe, voir les développements que propose Rudi Dutschke sur le rapport entre économie et idéologie autour de la question de la praxis révolutionnaire, in U. Bergmann, R. Dutschke, W. Lefèvre, B. Rabehl, La révolte des étudiants allemands (1968), Paris, Gallimard, 1968, p. 90 sq.

${ }^{35}$ H. MARCuSE, Vers la libération, op. cit., p. 83. 
Deuxièmement, j'aurais affirmé que ce que nous appelons en Amérique les hippies et ce que vous appelez les beatniks constitue la nouvelle classe révolutionnaire. Je n'ai jamais rien prétendu de ce genre. Je voulais simplement dire qu'il y a aujourd'hui dans la société des tendances - des tendances anarchiques, désorganisées, spontanées - qui annoncent une rupture totale avec les besoins de la société répressive. [Ces groupes] dénotent un état de désintégration l'intérieur du système : le phénomène en lui-même n'est pas encore vraiment une force révolutionnaire, mais il pourra peut-être jouer un rôle le jour où il sera relié à d'autres forces objectives beaucoup plus fortes. ${ }^{36}$

Comme la scission politique est externe et non plus interne, comme elle passe entre divers groupes et non à l'intérieur d'une même classe, le type de rapport entre les deux pôles du sujet révolutionnaire se trouve corrélativement modifié. Car il ne s'agit pas de conscientiser une masse ouvrière, il n'est pas question d'actualiser une virtualité révolutionnaire par le biais d'un appareil politique. Il s'agit au contraire de connecter deux groupes, de relier deux facteurs dissociés sans la médiation d'un organe représentatif. La scission entre un terme virtuel (classe en soi, masse passive et intégrée) et un terme actuel (classe pour soi, conscience active et révolutionnaire) se transforme en distance entre différents groupes sociaux dont l'un n'est pas l'actualisation de l'autre. Les étudiants ne représentent pas les ouvriers sur la scène politique (ils n'en sont pas la forme réalisée), pas plus que les ouvriers n'incarnent pratiquement la spontanéité de la conscience théorique estudiantine (ils n'en sont pas la force effectuée). Par conséquent, la possibilité d'une révolution dépend de la rencontre entre ces groupes sociaux, de la fécondation de la masse ouvrière par une conscience révolutionnaire impuissante, de la catalyse de la première par la seconde. On trouve chez Marcuse tout un champ lexical censé circonscrire le nouveau rapport éventuel entre les différents pôles du sujet révolutionnaire, non plus comme rapport de représentation et d'unification, voire de subordination, mais comme rapport de connexion qui maintient l'hétérogénéité des parties: «catalyse », «étincelle », " fécondation», «fermentation », « rencontre », « convergence », « collaboration », « lien », « coïncidence », « synchronisation »... Autant de termes qui ne sont pas des notions, et encore moins des concepts, mais qui orientent relativement clairement l'analyse marcusienne de la signification historique et générale des nouvelles formes d'opposition. Dans un texte de

${ }^{36}$ H. MARCuSE, La fin de l'utopie, op. cit., p. 17. 
1975 intitulé «Échec de la nouvelle gauche », Marcuse synthétise cet effort théorique en qualifiant d'une part la classe ouvrière (le facteur objectif) de « support » de la transformation sociale et, d'autre part, la révolte contre tout le type d'existence imposé par le système capitaliste - et non pas seulement contre la misère matérielle de l'ère industrielle, c'est-à-dire contre la paupérisation - (le facteur subjectif) de «moteur» de cette même transformation $^{37}$. Ainsi, la naissance des révoltes étudiantes ouvre un champ problématique en même temps qu'il légitime un espoir: celui de la coïncidence des deux facteurs de la révolution. Ce champ problématique se réfléchit tout entier dans la question de la stratégie révolutionnaire, c'est-à-dire de l'organisation des luttes.

\section{Théorie et pratique : la question de l'organisation}

La nouvelle figure du sujet révolutionnaire - figure brisée ou sujet scindé - légitime un espoir de transformation sociale puisque les révoltes étudiantes de la seconde moitié des années 1960 manifestent que la conscience révolutionnaire, éteinte dans la classe ouvrière (cf. les textes de 1964), renaît dans la jeune intelligentsia des pays du centre. Mais avec cet espoir naît une inquiétude: que ces deux groupes demeurent isolés l'un de l'autre. De manière générale, le problème de l'organisation se pose en fonction d'une double impossibilité : impossibilité de ne pas organiser la lutte, impossibilité de l'organiser selon ses formes traditionnelles.

Il est insensé de croire que la puissance du capitalisme, si hautement organisée et concentrée, peut être combattue en faisant l'économie d'une contre-organisation. Mais il est tout aussi insensé - ce qui veut dire : contredit par les faits - de penser qu'il soit possible de reprendre purement et simplement les formes traditionnelles d'organisation, centralisées et bureaucratiques: elles se sont désormais trop profondément intégrées à l'état de choses qu'il s'agit de renverser. ${ }^{38}$

Au niveau des révoltes étudiantes, cette double impossibilité se réfracte dans deux séries de problèmes :

${ }^{37}$ Cf. H. MArcuse, Actuels, « Échec de la nouvelle gauche » (1975), Paris, Galilée, 1976, p. 6-27.

${ }^{38}$ H. MARCuSE, Actuels, « Théorie et pratique » (1974), p. 83-84. 
En premier lieu, la question de l'organisation des étudiants est confrontée à la question de leur connexion aux forces traditionnelles de la transformation sociale que sont les masses ouvrières. Envisager la tâche des mouvements politiques d'opposition en termes de connexion, de catalyse, de rencontre ou encore de synchronisation, c'est dire a contrario que la situation actuelle de la lutte est marquée par une coupure, voire par une série de coupures : les étudiants coupés des masses prolétariennes, ces mêmes masses coupées de la conscience adéquate de leur état et de leurs intérêts, la population des ghettos coupées des formes nécessaires d'organisation. Plus largement, l'idée d'une coupure entre étudiants et ouvriers pose la question de la portée politique générale des revendications «étudiantes » énoncées depuis l'appareil universitaire ${ }^{39}$ ainsi que de leur capacité à initier une subjectivation de l'ensemble du champ social, c'est-à-dire à transformer la situation prérévolutionnaire en situation révolutionnaire ${ }^{40}$. En quel sens peut-on dès lors dire que la jeunesse étudiante, dans sa fonction de critique du champ social, ou bien porte une revendication de nature universelle, ou bien manifeste que l'écart entre théorie et pratique reste infranchissable et que la soudure des deux ordres reste suspendue ? Cette question prend sa pleine mesure dans le rapport entre les luttes étudiantes et les mouvements tiers-mondistes et dans la solidarité qui peut naître entre les différentes forces subversives dispersées sur la planète.

${ }^{39}$ Cf. H. MARCuSE, Vers la libération, op. cit., p. 82 : «"Le mouvement étudiant” - ce terme est déjà en lui-même idéologique et dérogatoire : il dissimule le fait que le mouvement est activement appuyé par de nombreux membres plus âgés de l'intelligentsia, et par d'importantes fractions de la population non étudiante. De plus, ce terme suggère des aspirations et des buts très différents de la réalité; les revendications générales pour une réforme du système d'enseignement ne font qu'exprimer des objectifs plus vastes et plus essentiels ».

${ }^{40}$ Cf. ibid., p. 79 : «La constellation qui prévaut dans les métropoles capitalistes nécessité objective d'un changement radical, et paralysie des masses - semble caractéristique d'une situation non pas révolutionnaire, mais prérévolutionnaire. Pour que la situation puisse devenir révolutionnaire, il faudrait que l'économie capitaliste mondiale vienne à s'affaiblir de façon critique, et que l'agitation politique gagne ne extension et en intensité ; alors, tout serait clair. C'est précisément de son rôle préparatoire que l'agitation politique tire sa signification historique : celle-ci consiste à développer chez les exploités la connaissance (consciente autant qu'inconsciente) grâce à laquelle leur existence pourrait s'affranchir des besoins asservissants qui perpétuent leur dépendance à l'égard du système d'exploitation. À défaut de cette rupture, qui ne peut être l'effet que d'une formation politique fondée sur l'action, les forces de rébellion, si élémentaires et immédiates soient-elles, risquent d'être écrasées, ou de devenir l'appui de masse de la contre-révolution ». 
Une autre série de problèmes découle directement de ce concept marcusien de «solidarité ». À ce concept incombe la fonction de théoriser le type spécifique de connexion ou d'articulation susceptible de naître entre ces différentes forces. On l'a vu, ce problème constitue l'alpha et l'oméga de la question des nouvelles modalités d'organisation. Or, le problème de la solidarité est particulièrement prégnant pour les mouvements étudiants, et notamment pour la révolte allemande d'abord parce que la situation de BerlinOuest cristallise en un même lieu le rapport entre conjoncture locale et nationale (les événements berlinois, la politique intérieure de la RFA) et situation internationale (Berlin comme « vitrine du monde libre »). Il l'est également dans la mesure où le mouvement étudiant n'est pas seul au sein de la Nouvelle Gauche, mais coexiste avec d'autres groupes de revendication : mouvements féministes, populations marginales des ghettos, luttes de libérations nationales dans le Tiers Monde. La multiplicité des groupes d'opposition confronte leur organisation à une tension constitutive mais non moins problématique: tension entre une tendance à l'unification homogénéisante et centralisatrice d'un côté (retour des tentations de subordination des revendications à des critères unilatéraux, au primat de la classe ouvrière, au dirigisme bureaucratique et à la forme-parti) et, d'un autre côté, une tendance à l'éclatement groupusculaire (cf. les exclusions de la SDS, notamment de la Kommune $I$ en 1967) menaçant d'instaurer une dérive individualiste voire fasciste (repli sur la dimension subjective, mais aussi morcellement et concurrence des luttes doublé d'un puissant vecteur identitaire).

Pour Marcuse, une des tâches principales qui attend les groupes d'opposition qui constituent la Nouvelle Gauche, et en particulier les mouvements étudiants, réside dans la synchronisation de ces groupes avec les masses ouvrières. En effet, un des dangers qui menace les luttes étudiantes est leur isolement sur l'échiquier politique, qui les ferait tourner à vide. C'est particulièrement le cas en Allemagne de l'Ouest où les étudiants doivent faire face à l'hostilité du prolétariat et des organisations ouvrières, savamment entretenue par la presse Springer (et la personnalisation de Dutschke comme «leader» du mouvement ${ }^{41}$ ). Pour Marcuse, sur l'échelle de l'écho des luttes étudiantes dans les masses ouvrières, l'Allemagne de l'Ouest se trouve tout en

${ }^{41}$ Cf. C. KohSER-Spohn, Mouvement étudiant et critique du fascisme en Allemagne dans les années soixante, op. cit., p. 214-216. 
bas, aux côtés des États-Unis :

Dans les pays fascistes et semi-fascistes, les militants étudiants - qui sont partout une minorité - trouvent un appui dans le prolétariat rural et industriel; en France et en Italie, ils ont réussi à obtenir une aide incertaine (et passagère !) des partis et unions d'une gauche puissante ; en Allemagne de l'Ouest et aux États-Unis, ils se heurtent à l'hostilité véhémente des "gens" et des organisations ouvrières. ${ }^{42}$

Que cette échelle puisse présenter tous ces degrés s'explique par la situation de bordure de l'Université comme de toute pensée critique dans la société, à l'articulation du théorique et du pratique, du savoir et de l'action politique, situation-limite qui autorise des perceptions contradictoires $\mathrm{du}$ même champ. Ainsi, la dynamique de fécondation du pratique par le théorique peut-elle se trouver bloquée par la prétendue «neutralité » de l'académie, de même que l'Université peut rapidement embraser le champ social (comme l'a montré le cas français, mais pas le cas allemand).

Ce qui apparaît aujourd'hui comme une "politisation" externe de l'Université par des éléments radicaux relève en réalité - comme si souvent dans le passé - de la dynamique interne, "logique», de l'enseignement: la connaissance se traduit en réalités, les valeurs humanistes en conditions humaines d'existence. (...) Le savoir n'est pas seulement transcendant (vis-à-vis du monde objectif, de la réalité) en un sens épistémologique : en tant qu'il s'oppose aux formes répressives de l'existence, il est politique. Par le refus de la liberté d'action politique à l'Université, on perpétue la coupure entre raison théorique et raison pratique, on restreint l'efficace et le champ d'action de l'intelligence. Ainsi, les revendications universitaires entrainent le mouvement au-delà de l'Université : vers les rues, les bidonvilles, la « communauté ». ${ }^{43}$

Dès lors, si les étudiants sont coupés des masses ${ }^{44}$, ce n'est pas en vertu de l'extériorité du savoir, mais à cause des forces contre-révolutionnaires qui ont tout intérêt à maintenir l'idée de la neutralité du champ académique, et le font - malgré les expériences de travail en milieu ouvrier ou en entreprise et les tentatives conseillistes initiées par les groupes marxistes-léninistes à la fin des années 1960 tentant de réorganiser un « mouvement ouvrier » (Opposition

${ }^{42}$ H. MARCuSE, Vers la libération, op. cit., p. 83.

${ }^{43}$ Ibid., p. 85.

${ }^{44}$ Sur ce point, cf. C. KoHSER-SPOHN, Mouvement étudiant et critique du fascisme en Allemagne dans les années soixante, op. cit., p. 225-262. 
Extra-Parlementaire [APO], Gauche Prolétarienne/Initiative de parti [PL/PI], Front Prolétarien $[\mathrm{PF}]$, Parti Communiste Allemand/Organisation en Construction [KPD/AO], Ligue Communiste/Marxistes-Léninistes [KB/ML]). Uwe Bergmann en résume l'idée quand il déclare au sujet des activités politiques au sein de l’Université de 1958 à 1964 :

le recteur ne condamnait les prises de position politiques du corps étudiant, que si elles contrevenaient à une ligne donnée. Invoquant le "principe de la neutralité" quand il s'agissait de réfugiés algériens, alors qu'il se laissait guider par l'opportunité quand il était question d'étudiants est-allemands, le rectorat revint à la "neutralité" politique pendant l'été 1963 lorsqu'il interdit une campagne de signatures contre le sort réservé à Hong-Kong aux réfugiés chinois. ${ }^{45}$

Chez Marcuse, l'isolement des étudiants, et plus généralement de tous les marginaux improductifs de la Nouvelle Gauche, ne fait pas l'objet d'un constat simplement pessimiste. Il est avant tout l'indice de la radicalité de la négativité historique à l'œuvre dans les années 1960, négativité qui menace tout le système et donc également la classe ouvrière intégrée :

loin de témoigner de l'absence de racines sociales du mouvement, cet isolement correspond à la situation historique actuelle; il traduit en vérité la "négation catégorique" de toute la culture du capitalisme monopoliste à son stade le plus avancé. Il manifeste l'hétérodoxie sans précédent de la révolution, comme contradiction radicale de la culture établie - y compris la culture de la classe ouvrière ${ }^{46}$

Mais que le fossé entre la Nouvelle Gauche et la classe ouvrière soit objectivement fondé n'enlève rien à la nécessité de leur jonction, comme « condition préalable du changement ${ }^{47}$. Une telle jonction, on l'a vu, n'est ni un rapport de représentation, de subordination, mais une rencontre des deux groupes «partant de leur propre base » et dans leur distance même ${ }^{48}$. Et ceci

${ }^{45}$ U. Bergmann, R. Dutschke, W. Lefèvre, B. Rabehl, La révolte des étudiants allemands, op. cit., p. 27.

${ }^{46}$ H. MARCusE, Contre-révolution et révolte, op. cit., p. 51.

${ }^{47}$ Ibid., p. 60.

${ }^{48} \mathrm{Cf}$. ibid., p. 60 : «La jonction des deux forces [est] une condition préalable du changement, la conscience syndicale doit se transformer en conscience politique, en conscience socialiste. Ce n'est pas en "allant aux ouvriers", en se joignant à leurs piquets de grève, en épousant leurs "causes", que l'on y parviendra. La jonction ne sera rendue possible que par un processus de changement social dans lequel chacun des deux groupes agira en partant de sa propre base, et en fonction de sa conscience, de ses doléances et de ses griefs propres. » 
pour deux raisons. Pour une raison organisationnelle d'abord, censée écarter un double danger: soit reproduire la forme-parti héritée de Lénine, soit sombrer dans un idéal spontanéiste victime de la fétichisation de la pratique des masses. Il existe aussi une autre raison, d'ordre historique ou conjoncturel : c'est que, dans les pays du centre, l'intégration de la classe ouvrière au système capitaliste a rendu tout bonnement impensable la création d'un parti révolutionnaire de masse; seule subsiste objectivement la possibilité d'échafauder des bases locales et décentralisées ${ }^{49}$. Néanmoins, il est remarquable que pour illustrer ses propos sur les tentatives de jonction entre étudiants et ouvriers, Marcuse cite les cas italien et français, mais non la révolte des étudiants allemands pour laquelle la rencontre avec la classe ouvrière a été un échec. Dès lors, si l'on peut trouver chez Marcuse un diagnostic de la spécificité du cas ouest-allemand, il faut probablement le déceler dans les blancs du texte : il existe en creux, dans le silence de l'analyse et dans l'absence de référence que Marcuse fait à l'Allemagne au moment même où il mentionne l'Italie et la France. Et de fait, force est de constater qu'en RFA la «catalyse » de la classe ouvrière par les étudiants n'a pas été produite, que l'« étincelle » n'a pas eu lieu. Aux yeux de Marcuse, cet échec n'est cependant pas que l'effet de la léthargie des masses ouvrières (ou de la seule presse Springer) : de manière générale, les étudiants sont eux-mêmes partiellement responsables de cet échec dans la mesure où leur fascination pour l'action prolétarienne de masse les a conduits à méconnaître que leur propre base constitue un point de départ potentiel pour une révolution globale.

Prisonnier de son fétichisme ouvriériste, le mouvement étudiant répugne à admettre, voire nie tout simplement qu'il a, sur les campus, la base qui lui appartient en propre dans l'infrastructure elle-même. En outre, cette base va des campus aux institutions politiques et économiques qui emploient de la "main-d'œuvre instruite". ${ }^{50}$

Témoigne de cette fétichisation ouvriériste la coexistence de deux tendances au sein du SDS, l'une orthodoxe ouvriériste ou traditionaliste (représentée par les présidents délégués Schauer et Dabrowski) et l'autre antiautoritaire (menée par Dutschke et consorts dès leur entrée au SDS), tendances dont la conflictualité éclate notamment au sujet du rôle du Vietnam

${ }^{49} \mathrm{Cf}$. H. MarcuSE, Actuels, «Échec de la nouvelle gauche », p. 29. 
pour et dans la lutte ${ }^{51}$.

Pour Marcuse, Rudi Dutschke a bien vu que le mouvement étudiant avait la possibilité de court-circuiter la fausse alternative entre revendications internes à l'Université et revendications politiques générales, du fait de la position spécifique des institutions académiques : l'Université comme lieu à la fois de production de la force de travail et de reproduction idéologique. Il s'agit là d'une des seules mentions que Marcuse fait du leader estudiantin dans ses écrits ${ }^{52}$. La référence à Dutschke intervient à un moment décisif de l'argumentation, lorsqu'il s'agit de penser l'articulation entre l'action au sein des institutions universitaires et en dehors d'elles :

Pour élargir la base du mouvement étudiant, Rudi Dutschke a proposé la stratégie de la « longue marche à travers les institutions » : il s'agit de travailler contre les institutions établies tout en $y$ travaillant, mais pas seulement de les saper de l'intérieur, plutôt au contraire de « faire son boulot », d'apprendre (à programmer et lire le langage des ordinateurs, à enseigner à tous les niveaux, à se servir des mass media, à organiser la production, à repérer l'obsolescence calculée et à y faire échec, à concevoir l'esthétique industrielle, etc.), et de préserver en même temps sa propre conscience en travaillant avec autrui. L'effort concerté pour instaurer des contre-institutions fait partie de cette longue marche. (...) J'ai souligné le rôle clé des universités dans la période actuelle : elles peuvent encore fonctionner en tant que centres de formations de contrecadres. La «restructuration» nécessaire au succès de cet objectif dépasse une participation étudiante résolue et un enseignement non autoritaire. Non, rendre l'Université «adaptée» au monde d'aujourd'hui et à celui de demain, c'est lui demander de mettre en relief les forces qui ont fait de la civilisation ce qu'elle est aujourd'hui et ce qu'elle pourrait être demain ; c'est cela, l'éducation politique. ${ }^{53}$

${ }^{51}$ Cf. C. KohSER-Spohn, Mouvement étudiant et critique du fascisme en Allemagne dans les années soixante, op. cit., p. 99-105.

${ }^{52} \mathrm{Cf}$. la lettre de Marcuse à Dutschke du 16 avril 1971 (reproduite in H. MARCUSE, Nachgelassene Schriften. Band 4: Die Studentenbewegung und ihre Folgen, p. 209-210).

${ }^{53}$ H. MARCuSE, Contre-révolution et révolte, op. cit., p. 78-79. Sur la question de l'élargissement et de la connexion de la lutte estudiantine aux autres sphères de la société, cf. R. DutschKe, «Les conditions historiques de la lutte internationale pour l'émancipation », in U. Bergmann, R. Dutschke, W. Lefèvre, B. Rabehl, La révolte des étudiants allemands, op. cit., p. 185 (position des étudiants dans le champ social) ; p. 194-195 (élargissement de la lutte) ; p. 196 (refus de la forme-parti) ; p. 197 (léthargie des masses ouvrières, dialectique de la prise de conscience des masses et séparation des facteurs objectifs et subjectifs). 
En RFA, la longue marche à travers les institutions est restée au stade programmatique, de même que l'hypothétique rencontre avec les ouvriers : le fossé entre théorie et pratique, comme entre praxis étudiante et praxis prolétarienne, n'a pas été franchi. À ce compte, et au regard de la tâche que lui avait prescrite Marcuse, la révolte des étudiants allemands semble avoir été un échec. C'est du moins une telle appréciation que l'on pourrait légitimement attendre de la part de Marcuse. Tel n'est pourtant pas le cas. Dans le texte d'une conférence tenue en 1975 et intitulée «Échec de la nouvelle gauche », on verra que Marcuse pose un diagnostic bien plus nuancé. Avant d'évoquer le bilan que tire Marcuse de la Nouvelle Gauche et de la révolte étudiante, il est nécessaire de comprendre la fonction assignée au Tiers Monde, et notamment au Vietnam, à l'égard des révoltes étudiantes.

Il n'est guère étonnant pour Marcuse que les étudiants aient eu beaucoup de mal à joindre leurs forces aux ouvriers, étant donné la marginalité sociale des premiers et le degré d'intégration des seconds. Toutefois, l'espoir et la tâche d'une synchronisation des forces subversives ne concernent ni uniquement ces deux groupes ni des échelles simplement locales ou nationales. Ainsi, pour Marcuse, le devenir révolutionnaire de la révolte des étudiants allemands ne doit pas être envisagé exclusivement à l'intérieur du capitalisme avancé pas plus qu'on ne peut compter sur les seules luttes de libération nationale dans le Tiers Monde pour une révolution mondiale. La synchronisation des forces doit avoir lieu entre le centre et la périphérie, entre les forces de subversion intérieures au système capitaliste et celles qui existent dans son espace d'expansion nécessaire :

c'est précisément à cause de cette relation interne entre les révolutions étrangères et les métropoles que persiste le lien fatal entre les perspectives de mouvements de libération et les perspectives de changement radical dans les métropoles : les forces de «négation » qui sont à l'œuvre à l'étranger doivent être synchronisées avec celles qui sont à l'œuvre à l'intérieur, et cette synchronisation ne peut jamais être le résultat de la simple organisation; elle doit avoir sa base objective dans le processus économique et politique du capitalisme corporatif. Ces facteurs objectifs s'annoncent dans les tensions et les à-coups de l'économie capitaliste. ${ }^{54}$

On l'a vu, synchronisation ne signifie pas assimilation mais jonction des

${ }^{54}$ H. MARCUSE, «Réexamen du concept de révolution », p. 27. Sur l'existence de forces subversives au centre et à la périphérie, cf. H. Marcuse, La fin de l'utopie, op. cit., p. 25-26. 
forces dans leur distance : « ce qui est à l'ordre du jour dans les métropoles et au Vietnam est différent, mais les deux choses peuvent être mises en relation $»^{55}$.

On sait que cela a précisément été le cas en 1965-1966 à la F.U., au cours de ce qu'Uwe Bergmann nomme le « semestre Vietnam ».

Nul événement politique n'a autant que la guerre du Vietnam joué un rôle décisif en ce qui concerne les discussions et la politisation des étudiants. Ce sont les préoccupations liées à cette guerre qui devaient provoquer le premier conflit important avec l'ordre régnant hors de l'Université. (...) Face à l'anéantissement de tout un peuple au nom de la démocratie, face à l'agression américaine contre le Vietnam, le mouvement étudiant trouva sa dynamique propre. ${ }^{56}$

Dans cette intrusion de la scène mondiale dans les débats et les discours de mobilisation étudiants (la guerre du Vietnam, mais aussi la visite de Moïse Tschombe en décembre 1964, les marches contre l'arme atomique de Pâques 1965, "l'attentat au pudding » contre le vice-président américain Humphrey en avril 1967, la manifestation à l'occasion de la visite du Shah d'Iran en mai 1967, etc.), dans la dialectique de politisation-dépolitisation relancée par cette intrusion, Guillaume Sibertin-Blanc avait vu l'occasion de forger le concept d' "événements mondiaux intérieurs ${ }^{57}$. Leur importance tient à ce que le court-circuit des scènes locale (l'Université) et internationale (exemplairement le Vietnam qui est «l'Espagne de notre génération ${ }^{58}$ ) produit un puissant effet de politisation qui décentre la lutte de son vis-à-vis étatique, transforme du même coup le système de positions qui détermine le champ politique de la lutte et met en échec les formes traditionnelles de la répression :

lorsque le processus de politisation étudiante, dans ses débats, dans ses mobiles, dans ses exigences et dans ses mots d'ordre, se trouve contaminé par les données de la conjoncture mondiale, la dépolitisation

${ }^{55}$ H. MARCUSE, La fin de l'utopie, op. cit., p. 31.

${ }^{56}$ U. Bergmann, R. Dutschke, W. LefĖvre, B. RaBehl, La révolte des étudiants allemands, op. cit., p. 36.

${ }^{57}$ Séminaire du GRM, séance du 26 septembre 2009: http://f.hypotheses.org/wpcontent/blogs.dir/1106/files/2013/01/GRM.3.Introduction..pdf

${ }^{58}$ «Le Vietnam est l'Espagne de notre génération » est un tract signé par des professeurs et intellectuels allemands en février 1968. 
devient particulièrement difficile, sinon impossible. ${ }^{59}$

Marcuse n'a pas forgé de conceptualité précise pour penser de tels effets de seuils au cours de la lutte: il n'en reste pas moins qu'il a constamment souligné l'importance de la conjoncture internationale, et notamment des luttes de libération nationale pour et dans les révoltes des pays du capitalisme avancé, et notamment les mouvements étudiants : il faut que «l'opposition étudiante [réussisse] à faire du Tiers Monde et de sa praxis révolutionnaire sa propre base de masse $»^{60}$.

L'importance des luttes dans le Tiers Monde à l'égard des révoltes dans les pays du centre ne les rend pas pour autant équivalentes quant à la perspective révolutionnaire. Pour Marcuse, en effet, leur synchronisation nécessaire ne supprime pas une certaine prévalence des pays du capitalisme avancé sur le Tiers Monde et de la production économique sur la conscience et l'idéologie : « la chaîne d'exploitation doit rompre à son maillon le plus fort », à savoir au niveau de la structure économique du capitalisme des métropoles ${ }^{61}$. Dans Vers la libération, Marcuse introduit une nette hiérarchie à l'intérieur de cette multiplicité de facteurs (facteurs subjectifs et objectifs, au centre et à la périphérie). Les facteurs subjectifs sont certes une condition nécessaire de la libération, mais ils restent dépendants de l'affaiblissement préalable des structures objectives du capitalisme, car seule la radicalisation de ses antagonismes est apte à engendrer un réveil des masses. D'une part, les luttes de libération nationale au sein du Tiers Monde n'ont de chance d'être efficaces au plan mondial qu'à condition que les pays du capitalisme avancés soient affaiblis, puisque ce sont ces derniers (notamment les Américains) qui y organisent et financent la contre-révolution (au Vietnam, à Cuba, en Amérique latine $)^{62}$. D'autre part, les révoltes dans les pays du centre produisent certes un

\footnotetext{
${ }^{59}$ Ibid., p. 23.
}

${ }^{60}$ H. MARCuSE, La fin de l'utopie, op. cit., p. 56-57, voir aussi ibid., p. 133.

${ }^{61}$ H. Marcuse, Vers la libération, op. cit., p. 108.

${ }^{62}$ Cf. ibid., p. 108 : «Seul l'affaiblissement interne des super-puissances pourra finalement les empêcher de financer et d'équiper la répression dans les pays les moins avancés. Les Fronts nationaux de libération constituent une menace pour l'existence de l'impérialisme; non seulement au niveau matériel, mais aussi sur le plan idéologique, ils représentent le catalyseur du changement. La révolution cubaine, le Vietcong, ont mis en évidence ce qu'il est possible de faire (...). Bien plus que de l' "humanisme socialiste" du jeune Marx, c'est de cette violente solidarité dans la défense, de ce socialisme élémentaire dans l'action, que le radicalisme de la Nouvelle Gauche a tiré sa forme et sa substance; ici encore (au niveau de l'idéologie), la révolution externe joue un rôle fondamental dans l'opposition intérieure des métropoles 
renouveau de la conscience révolutionnaire, instillent une « dissolution de la moralité sociale » en opposant un refus de la répression idéologique non moins que matérielle (économique et politique) ; mais leur efficacité dépend elle aussi de l'affaiblissement interne des économies nationales, comme le montre la paralysie des masses ${ }^{63}$. Marcuse confère ainsi in fine un certain privilège à l'économique sur l'idéologique et au centre sur la périphérie. Mais cette hiérarchie est encore trop schématique, car pour Marcuse les luttes de libération nationales du Tiers Monde ont une spécificité que n'ont pas les révoltes étudiantes : y coïncident les facteurs objectifs et subjectifs de la révolution, puisque les producteurs immédiats (majoritairement agricoles) participent également aux luttes. Le sujet révolutionnaire y est tendanciellement unifié, ou plutôt il n'y est pas encore scindé comme il l'est dans les pays du centre.

En effet, dans les sociétés capitalistes avancées, les modalités de la lutte prennent un relief particulier en raison de cette scission interne du sujet historique de la révolution. Celui-ci est deux fois dispersé : il est d'abord séparé en facteurs subjectifs et objectifs, on l'a vu ; mais le facteur subjectif est lui-même scindé en divers groupes hétéroclites, ceux qui composent la Nouvelle Gauche. Les mouvements féministes avancent ainsi des revendications qui, si elles ne sont pas incompatibles avec celles des étudiants (loin de là), n'y sont pas pour autant homogènes - de même pour la population des ghettos ${ }^{64}$. Chez Marcuse, cette dispersion des subjectivités militantes dans l'espace social fait l'objet d'un double constat, par rapport auquel il est possible de situer la position des étudiants. Premier constat : le morcellement a pour effet positif de rendre possible de nouvelles formes d'organisation non centralisées, sans pour autant qu'elles perdent un caractère « totalisateur ». Ces nouvelles formes d'organisation décentralisées, diffuses et relativement spontanées, outre qu'elles répondent à la perte de confiance envers les partis

capitalistes. Toutefois, cette force exemplaire, cette puissance idéologique de la révolution extérieure ne peuvent porter ses fruits que si le système capitaliste commence à perdre sa structure interne et sa cohésion ».

${ }^{63}$ Cf. ibid., p. 111 : «Certes, il s'agit là de facteurs subjectifs, mais qui pourraient avoir une efficacité matérielle s'ils s'ajoutaient aux tensions objectives, de nature économique et politique, auxquelles le système devra faire face à l'échelle mondiale. Alors, et seulement alors, se constituerait un climat politique dans lequel un appui de masse pourrait s'offrir aux nouvelles formes d'organisation qui seront nécessaires pour diriger le combat ».

${ }^{64}$ Sur la question des mouvements féministes chez Marcuse, cf. H. MARCuSE, Actuels, « Marxisme et féminisme » (1974), p. 37-58. 
traditionnels et à l'égard de toute forme d'autorité, permettent de tenir tête à la violence potentielle de la machine répressive d'État : ou plutôt de ne pas lui tenir tête, c'est-à-dire de ne pas entrer dans une opposition frontale avec lui. La rupture avec la forme-parti va dès lors de pair avec la transformation des objectifs de la lutte : il n'est plus question de s'emparer du pouvoir d'État (dont la puissance militaire et policière est trop concentrée) avec l'appui d'une action de masse (que la léthargie rend conservatrice, ou au mieux réformiste $)^{65}$. Cette diffusion de la contestation dans le champ social n'est pas pour autant l'indice d'une démission ou d'un manque d'ambition. Certes, le mouvement étudiant, comme la plupart des membres de la Nouvelle Gauche, est le fait de groupes minoritaires situés à la marge : il est de fait susceptible d'être soit récupéré soit isolé. Mais il a toutefois par ce fait même profondément renouvelé l'idée et la possibilité de la révolution comme transformation totale de l'ordre établi. En effet, si ces groupes sont à la marge, c'est parce qu'ils contestent la société existante dans ses dimensions tant objectives et économiques que subjectives et psychiques (cf. la composante esthétique de la révolte berlinoise avec le groupe Spur). Ainsi, les étudiants, malgré les problèmes stratégiques d'organisation et de lutte qu'ils rencontrent ont redonné vie à l'espoir d'un changement qualitatif et total (et non pas quantitatif, c'est-à-dire par le simple accroissement économique des forces productives). La révolution sera culturelle ou ne sera pas ${ }^{66}$. Que la révolte ait une dimension psychique et libidinale autant qu'économique ressort d'abord des conceptions originelles du groupe Subversive Aktion (« une révolte totale dans tous les domaines de la vie $\|^{67}$ ), mais plus encore de la critique que Dutschke fera du caractère encore trop « existentialiste » et pas assez « révolutionnaire » de la subversion menée par ce groupe ${ }^{68}$.

Second constat: 1'anarchisme et le spontanéisme qui caractérisent les nouvelles formes d'opposition restent tout de même exposés au danger majeur de l'inefficacité, mais aussi du repli identitaire et de l'usage extrême de la violence (cf. le mot malheureux de Habermas sur le « fascisme de gauche »). On sait que les tendances anarchistes ou spontanéistes de certaines luttes ont

${ }^{65} \mathrm{Cf}$. H. MARcuse, Contre-révolution et révolte, op. cit., p. 63-64.

${ }^{66} \mathrm{Cf}$. H. MARcuse, Actuels, p. 17, 22, 27, 30.

${ }^{67}$ "Eschatologisches Programm», Unverbindliche Richtlinien, 1, décembre 1962, Odensee/Danemark, p. 24, cité in C. KOHSER-SpoHn, Mouvement étudiant et critique du fascisme en Allemagne dans les années soixante, op. cit., p. 93.

${ }^{68}$ Cf. C. KoHSER-SPOHn, op. cit., p. 96. 
radicalement renouvelé leurs modalités mêmes (cf. la "manifestation promenade » de décembre 1966 à Berlin). De même, l'ancrage anti-autoritaire du mouvement allemand crée un débat au sein du SDS sur le sens et l'intérêt stratégique du parlementarisme et, plus généralement, de l'utilisation des institutions démocratiques dans le combat politique (d'où l'ambivalence entre deux conceptions de la démocratie, bourgeoise-formelle ou directeconseilliste) ${ }^{69}$. Chez Marcuse, le constat que la Nouvelle Gauche rompt avec les organisations militantes centralisées et bureaucratiques traditionnelles se double de l'inquiétude que le manque d'organisation et d'éducation des subjectivités militantes soit tout bonnement inefficace : quoi de pire pour un penseur critique qu'une spontanéité non éduquée ? D'où chez Marcuse la tentative de concilier spontanéité et autorité, ou éducation. Comment éviter le piège de la spontanéité sans retomber dans celui du dirigisme?

La spontanéité n'est pas antagoniste de l'autorité ; dans la mesure où la pratique révolutionnaire est explosion de besoins vitaux qui, on l'a vu, ne portent pas nécessairement sur les nécessités matérielles de la vie, elle plonge ses racines dans la spontanéité ; mais cette spontanéité peut être trompeuse, elle peut résulter de l'introjection de besoins sociaux suscités par l'ordre établi mais contraires à la libération de l'existence humaine. L'endoctrinement et la manipulation intenses des gens appellent une contre-éducation et une organisation intenses en défense. Or cette nécessité se heurte aux tendances antiautoritaires de la Nouvelle Gauche. ${ }^{70}$

Ainsi, la situation actuelle est marquée par un double écueil : d'un côté, l'opposition historique aux partis de masse bureaucratiques et dirigistes est légitime, de l'autre elle paraît condamner l'efficacité de la lutte. Cette tension constitutive de la Nouvelle Gauche « entre rébellion personnelle et rébellion politique, entre libération privée et révolution sociale $»^{71}$, rejoint le second

${ }^{69} \mathrm{Cf}$. ibid., Deuxième partie, chap. III : «La démocratie directe contre la démocratie bourgeoise ». S'inspirant de la démocratie directe héritée du conseillisme allemand, Dutschke affirme ainsi : « Lorsque nous nous disons extraparlementaire, cela veut dire que nous visons un système de démocratie directe, c'est-à-dire une démocratie de conseils qui permette aux hommes à la conscience critique d'élire et de déposer leurs délégués lorsque cela leur semble nécessaire », " Gespräch mit R. Dutschke », Der Spiegel, 21, 29, 1967, cité par C. KoHSERSpoHn, Mouvement étudiant et critique du fascisme en Allemagne dans les années soixante, op. cit., p. 152.

${ }^{70}$ H. MARcuse, Contre-révolution et révolte, op. cit., p. 69.

${ }^{71}$ Ibid., p. 70. 
danger qui menace, aux yeux de Marcuse, son efficacité et à la limite son existence : le repli des luttes sur la libération individuelle marqué par un refus de toute éducation ou théorie. C'est exemplairement le cas de la Kommune I et de son rapport au SDS, dont il sera exclu en mai 1967 : est-ce l'expérience d'un nouveau mode de vie révolutionnaire ou une progressive dérive apolitique ? En témoigne le célèbre mot du communard Kunzelmann : "Que m'importe le Vietnam, j'ai des problèmes d'orgasme ! ».

Il est vrai que là se situe précisément ce qui empêche la révolte d'avoir son plein effet; les «contre-cultures » créées par la nouvelle gauche perdirent leur impact politique et se détruisirent elles-mêmes en revenant à une sorte de « libération » individuelle (la drogue, le culte du gourou et autres sectes pseudo-religieuses), en professant un antiautoritarisme abstrait doublé de mépris pour la théorie en tant que guide de la pratique. ${ }^{72}$

Mais si l'on persiste à faire une lecture symptomale du texte de Marcuse pour y déceler un diagnostic en négatif de la révolte berlinoise, on conçoit que la situation de l'Université est à cet égard relativement préservée de ce danger, et ce constitutivement, $d u$ fait de son caractère institutionnel et de sa position dans le champ social à la frontière de l'individuel et du collectif, du psychique et du matériel, de l'idéologique et de l'économique. Mais on n'est pas étudiant toute sa vie... En revanche, en dehors de l'Université, l'anti-autoritarisme a également donné lieu à des formes violentes de lutte (notamment avec la RAF en Allemagne). Or, lorsqu'il est question de l'usage de la violence dans le combat politique, Marcuse évoque rarement cette «bascule» possible de l'anti-autoritarisme en lutte armée ; il en reste le plus souvent à une opposition entre « violence de l'oppression » et « violence de la libération », d'agression ou de défense, violence institutionnalisée et droit de résistance ${ }^{73}$. À cet égard, l'usage de la violence dans la confrontation aux autorités «demeure donc avant tout un problème de tactique $\rangle^{74}$ et non pas un problème quant au devenir du combat politique. Cependant, la question du «terrorisme » révolutionnaire, de la tactique de guérilla, pose non seulement un problème de légalité juridique, mais interroge aussi - et peut-être surtout - les tendances internes des organisations et des formes de la lutte. La possibilité d'un

\footnotetext{
${ }^{72}$ H. Marcuse, Actuels, op. cit., p. 19.

${ }^{73}$ Cf. H. Marcuse, La fin de l'utopie, op. cit., p. 49-50.

${ }^{74}$ Ibid., p. 50.
} 
« fascisme de gauche » (selon le mot de Habermas), c'est-à-dire en réalité l'ambivalence de l'usage de la violence révolutionnaire oscillant entre deux pôles extrêmes (libération et terrorisme), n'est dès lors jamais véritablement prise en compte par Marcuse (et ce au-delà du fait que les signifiants « fascisme » et " néo-fascisme » désignent chez lui uniquement la violence contre-révolutionnaire).

\section{Déplacement ou continuité ?}

De quelle nature est le déplacement théorique opéré par Marcuse, à l'occasion de l'analyse des révoltes étudiantes, quant à la conception marxiste du sujet révolutionnaire ? On pourrait considérer que ce déplacement masque en réalité une continuité souterraine.

Il y a bel et bien, à la faveur des mouvements étudiants que Marcuse s'est efforcé de théoriser (surtout le mouvement américain, un peu les mouvements allemand, français et italien) un déplacement théorique majeur, qui se réfléchit tout entier dans sa compréhension de la signification historique de la Nouvelle Gauche. Comme l'indique explicitement le texte « Réexamen du concept de révolution », celle-ci symbolise une troisième voie marquée par le refus simultané de la voie capitaliste et de la voie socialiste. Replacée dans le contexte propre aux pays du centre marqués par une intégration idéologique de la classe ouvrière, la Nouvelle Gauche, et notamment la jeunesse étudiante militante, apparaît comme la renaissance du facteur subjectif de la révolution, qui s'était absenté du prolétariat et de ses organes représentatifs. La nouveauté théorique de l'analyse de Marcuse repose ainsi sur la nouvelle forme de scission qui existe entre l'en-soi et le pour-soi de la transformation sociale. Il n'incombe plus au parti marxiste-léniniste d'éduquer les masses mais aux étudiants de les féconder, de les catalyser, de les réveiller, bref de les sortir de leur léthargie conservatrice ou réformiste. C'est le sens de la distinction que fait Marcuse entre le support et le moteur de la révolution : le support est puissant mais paralysé, le moteur est actif mais sans base de masse - d'où l'urgence du « renforcement numérique $\rangle^{75}$, c'est-à-dire de la jonction de leurs forces respectives.

Cependant, si elle déplace la distinction entre une masse prolétarienne

${ }^{75}$ H. MARcuse, Contre-révolution et révolte, op. cit., p. 56. 
et une avant-garde consciente censée la conscientiser, le rapport entre support et moteur de la transformation sociale reproduit le présupposé de cette distinction ou son modèle implicite : l'opposition entre une matière passive et une forme active, que celle-ci soit logée dans le parti marxiste-léniniste comme médiation interne à la classe ouvrière ou qu'elle s'incarne dans un groupe social (relativement) extérieur à celle-ci : les étudiants. Avec l'idée d'une classe révolutionnaire en soi, ou d'un sujet objectif de l'émancipation (objectif mais passif ou virtuel), Marcuse réactive le schéma hylémorphique, qui prend d'abord son sens dans le cadre d'une théorie de la connaissance liée à un principe de hiérarchisation socio-technique du travail ${ }^{76}$. On retrouve un tel schéma hylémorphique dans le rapport faussement égalitaire de la distinction entre théorie et pratique (la théorie comme "guide» de la pratique), dont on peut observer les effets dans la théorie marcusienne de l'organisation (la nécessaire éducation de la spontanéité). Marcuse demeure in fine incapable de s'affranchir réellement de la fausse alternative dialectique de l'autonomie et du centralisme, de la spontanéité des masses et de la réflexivité théorique des avant-gardes, puisque la question de l'organisation de la contestation reste irrémédiablement prise dans l'horizon d'une unification, d'une totalisation ou d'une synchronisation possibles et à venir, où viendraient se résoudre l'ensemble des contradictions qui travaillent le champ social ${ }^{77}$.

${ }^{76}$ Sur ce point, cf. G. Simondon, L'individuation à la lumière des notions de forme et d'information, Grenoble, Million, 2005, p. 51 : «Ce que le schéma hylémorphique reflète en premier lieu, c'est une représentation socialisée du travail et une représentation également socialisée de l'être vivant individuel (...). L'opération technique qui impose une forme à une matière passive et indéterminée (...) est essentiellement l'opération commandée par l'homme libre et exécutée par l'esclave (...). Le caractère actif de la forme, le caractère passif de la matière, répondent aux conditions de la transmission de l'ordre qui suppose une hiérarchie sociale : c'est dans le contenu de l'ordre que l'indication de la matière est un indéterminé alors que la forme est détermination, exprimable et logique ».

${ }^{77}$ On sait que c'est sur une toute autre voie que s'engageait Althusser à la même époque dans Pour Marx et Lire le Capital, en cherchant à soustraire la conceptualité marxiste de l'idéalisme hégélien, inaugurant une voie divergente dans le champ marxiste (dont se ressentent par exemple les outils théoriques à même de produire des analyses de conjonctures). Il semble bien que le champ théorique actuel obéisse encore à cette divergence de voies, en ce qu'il est schématiquement scindé en une tradition critique inspirée de l'École de Francfort (dont la « philosophie sociale» ou «critique » serait aujourd'hui le fer de lance) et une tradition althussérienne (dont Balibar, Foucault, Deleuze-Guattari et ceux qui s'en réclament aujourd'hui seraient les héritiers principaux). Les usages actuels de Marx témoignent de l'existence de ces deux courants (bifurcation qui n'interdit pourtant pas certaines passerelles théoriques) : il ne viendrait par exemple pas plus à l'idée des héritiers de la tradition critique d'abandonner le 
Il nous semble que la casuistique marcusienne à propos du nouveau sujet révolutionnaire, casuistique dont témoignent les distinctions incertaines et des termes parfois peu conceptualisés, renvoie en dernière analyse à une difficulté constitutive du moment historique de l'après-guerre, dont les conséquences sont observables dans la théorie. Guillaume Sibertin-Blanc en faisait la remarque, en généralisant le diagnostic que Badiou faisait du mai 68 en France : on assiste à cette époque à la dissipation corrélative et de l'agent objectif de l'émancipation inscrit dans la réalité sociale et de la conviction que cet agent objectif doit être transformé en puissance subjective.

Disons que si tend à vaciller la représentation d'un sujet de l'action transformatrice radicale qui sera préfiguré, prédéterminé, ou d'une manière ou d'une autre pré-donné dans l'objectivité sociale, ce n'est pas parce que cette représentation elle-même disparaît, mais plutôt parce qu'elle se complexifie et se démultiplie (...), et parce que c'est corrélativement l'objectivité sociale elle-même ou ce qui serait représentable comme tel qui vacille. ${ }^{78}$

Les acrobaties théoriques auxquelles est contraint Marcuse pour tenter de ménager à la fois les analyses de conjoncture des mouvements étudiants de la fin des années 1960 d'un côté et la dialectique hégéliano-marxiste à l'œuvre dans la théorie critique ne doivent pas nous tromper : leur conciliation est plus que problématique. $\mathrm{Au}$ fur et à mesure qu'il constate la " désespérante dispersion » de l'agent de la transformation sociale dans les différentes couches sociales ainsi que dans les différentes régions du monde, vacille la possibilité d'identifier et d'articuler les diverses figures du négatif, qui ne sont pourtant rien d'autre que le moteur de la dialectique historique. Dès lors, la conjoncture étudiante vient révéler à la théorie ce que Balibar considère être la non-coïncidence constitutive du prolétariat avec lui-même, son caractère fondamentalement scindé entre l'en-soi et le pour-soi, entre l'existence objective économique et matérielle de classe d'une part et la conscience subjective d'une praxis de masse d'autre part ${ }^{79}$. Il n'est pas sûr,

concept dialectique d'aliénation (et les textes de Marx qui en usent) qu'aux post-althussériens de l'utiliser (du moins en son sens fort).

${ }^{78}$ Séminaire du GRM, séance du 26 septembre 2009, op. cit.

${ }^{79} \mathrm{Cf}$. E. Balibar, La crainte des masses, « Le prolétariat insaisissable », Paris, Galilée, 1997, p. 244-248 : «si, dans Le Capital, le prolétariat est concrètement présent, mais sans signifiant unique, c'est surtout qu'il intervient selon deux modalités qui ne sont pas purement et simplement superposables (...) : à la fois comme classe et comme masse. (...) Le fait que le 
pourtant, que Marcuse tire toutes les conséquences de cette non-coïncidence fondamentale, étant donné l'importance qu'il accorde à la possibilité de synchronisation des forces subversives, c'est-à-dire à l'espoir de leur unification finale. Cette tension dans le corpus marcusien manifeste une tension entre l'idée hégélienne de résolution dialectique réelle des contradictions et l'analyse d'une conjoncture qui met en échec non pas l'unification éventuelle et provisoire des contradictoires mais la garantie de cette unification. Comme l'écrit Balibar :

Poser que l'émergence d'une forme de subjectivité (ou d'identité) révolutionnaire est toujours un effet partiel, jamais une propriété de nature, et ne comporte par conséquent aucune garantie, c'est s'obliger à rechercher les conditions qui, dans la conjoncture, peuvent précipiter des luttes de classes en mouvements de masses, et les formes de représentation collective qui, dans ces conditions, peuvent maintenir dans les mouvements de masses l'instance de la lutte des classes. Rien ne dit, bien au contraire, que ces formes soient éternellement les mêmes... ${ }^{80}$

En réalité, Marcuse reste sensible à ce risque dans la mesure même où il insiste sur la puissance de la contre-révolution fasciste susceptible de mettre fin aux tendances et aux espoirs révolutionnaires :

Un danger menace pourtant la nouvelle gauche, comme la gauche dans son ensemble: celui d'être victime des tendances réactionnairesagressives du capitalisme avancé. Ces tendances se renforcent à mesure que la crise s'étend et qu'elle oblige le système à chercher une issue dans la mesure d'une nouvelle guerre et dans la répression de l'opposition. Une nouvelle fois le fascisme menace de barrer la route au socialisme. L'alternative classique "socialisme ou barbarie" est aujourd'hui plus actuelle que jamais. ${ }^{81}$

En revanche, Marcuse ne prend pas véritablement la mesure d'un autre risque qu'il ne mentionne que sporadiquement: la dérive groupusculaire,

prolétariat, qui est à la fois "classe" et "masse", ne soit pas un sujet donné, qu'il ne coïncide jamais avec lui-même - qu'il soit en quelque sorte ontologiquement dissocié -, ne signifie pas qu'il ne lui arrive jamais de se présenter et d'agir comme sujet dans l'histoire. Mais cette action révolutionnaire est toujours liée à une conjoncture, durable ou non, et n'existe que dans les limites qu'elle prescrit ».

${ }^{80}$ Ibid., p. 248.

${ }^{81}$ H. Marcuse, Actuels, « Échec de la nouvelle gauche », p. 34-35. 
«terroriste » ou «fasciste » des mouvements de contestation, dont l'Allemagne (comme l'Italie) sera le théâtre avec la Rote Armee Fraktion (RAF).

Dernière remarque, quant à la spécificité de la conjoncture allemande vis-à-vis des autres mouvements étudiants, en Europe et aux États-Unis. La révolte des étudiants berlinois, ne donne pas seulement à voir cette scission du sujet révolutionnaire, notamment à travers les problèmes très concrets des rapports de l'Université au champ social et du caractère politique de leurs revendications (cf. le rôle du recteur de la F.U. et de la presse Springer). Elle manifeste également de manière privilégiée un double phénomène inédit : le rôle de la conjoncture internationale dans la mobilisation et la politisation des subjectivités militantes doublé d'un mouvement antiautoritaire dont la radicalité est une réaction au passé fasciste de l'Allemagne (sur la réaction au passé fasciste, cf. les Ringvorlesungen dans les universités). L'insularité de Berlin et de sa mentalité pendant les années 1960 a probablement joué un rôle décisif dans la radicalisation des antagonismes. En effet, pour Kohser-Spohn,

Si la mentalité antiautoritaire s'était essentiellement développée à Berlin, c'est en raison de la situation géopolitique de la ville. La situation insulaire de Berlin et l'histoire récente de la ville (blocus de 1948, construction du Mur en 1961, difficultés économiques...) y avaient développé une idéologie et une mentalité de ghetto, qui se manifestaient par un sentiment communautaire exacerbé, s'exprimant dans la même volonté de "tenir bon" et de montrer aux "frères et sœurs" de l'Est les bienfaits de la démocratie. L'esprit berlinois était entièrement canalisé vers la critique du communisme que corroborait le traumatisme de l'invasion "russe". La conscience de la fragilité géopolitique de la ville et de la communauté sans cesse menacée poussait les Berlinois à ne tolérer aucune critique qui pût mettre en danger "la vitrine du monde libre". Aussi étaient-ils plus prompts qu'ailleurs à sanctionner des comportements qui déviaient de la norme communautaire. $^{82}$

La position singulière de Berlin en Allemagne, en Europe et dans le monde court-circuite du même coup beaucoup plus facilement la conception stato-centrée de la révolte, de ses stratégies et de sa répression possible. Berlin

${ }^{82}$ C. KoHSER-Spohn, Mouvement étudiant et critique du fascisme en Allemagne dans les années soixante, op. cit., p. 107-108; sur la singularité de Berlin, voir le chapitre IX : «Le paradigme de Berlin ». 
est en quelque sorte un précipité des différentes échelles : locale (l'université et le pouvoir municipal), nationale (le gouvernement de la RFA) et internationale (la politique américaine à Berlin), ce dont les membres du SDS/Berlin seront conscients dès le milieu des années 1960. « Le consensus entre les autorités de la ville et celles de l'université révélait au SDS/Berlin que Berlin était le dernier endroit au monde où la population s'identifiait presque entièrement à la politique des USA, du fait de sa position géopolitique. Mais à cause de cette situation géographique, Berlin constituait un point névralgique pour la politique intérieure des États-Unis. Car si les intellectuels berlinois descendaient dans la rue pour protester contre la guerre américaine au Vietnam, les cercles conservateurs américains se sentiraient abandonnés par leurs derniers amis $\rangle^{83}$. Cela ne fut évidemment pas le cas en France, en Italie et, a fortiori, aux États-Unis. Ce fut seulement le cas en Allemagne. Il est alors frappant de constater que le diagnostic porté par Marcuse sur les révoltes estudiantines, qui envisage pourtant le cas allemand à travers une focale américano-centrée, n'ait pas tenu compte de ce trait profondément singulier.

${ }^{83}$ Ibid., p. 99-100. 